\title{
Noise-enhanced coupling between two oscillators with long-term plasticity
}

\author{
Leonhard Lücken, ${ }^{1}$ Oleksandr V. Popovych, ${ }^{2}$ Peter A. Tass, ${ }^{2,3,4}$ and Serhiy Yanchuk ${ }^{1,5}$ \\ ${ }^{1}$ Weierstrass Institute for Applied Analysis and Stochastics, Berlin, Germany \\ ${ }^{2}$ Institute of Neuroscience and Medicine - Neuromodulation, Jülich Research Center, Jülich, Germany \\ ${ }^{3}$ Department of Neurosurgery, Stanford University, Stanford, California, USA \\ ${ }^{4}$ Department of Neuromodulation, University of Cologne, Cologne, Germany \\ ${ }^{5}$ Institute of Mathematics, Technical University of Berlin, Berlin, Germany
}

(Received 30 November 2015; revised manuscript received 1 February 2016; published 8 March 2016)

\begin{abstract}
Spike timing-dependent plasticity is a fundamental adaptation mechanism of the nervous system. It induces structural changes of synaptic connectivity by regulation of coupling strengths between individual cells depending on their spiking behavior. As a biophysical process its functioning is constantly subjected to natural fluctuations. We study theoretically the influence of noise on a microscopic level by considering only two coupled neurons. Adopting a phase description for the neurons we derive a two-dimensional system which describes the averaged dynamics of the coupling strengths. We show that a multistability of several coupling configurations is possible, where some configurations are not found in systems without noise. Intriguingly, it is possible that a strong bidirectional coupling, which is not present in the noise-free situation, can be stabilized by the noise. This means that increased noise, which is normally expected to desynchronize the neurons, can be the reason for an antagonistic response of the system, which organizes itself into a state of stronger coupling and counteracts the impact of noise. This mechanism, as well as a high potential for multistability, is also demonstrated numerically for a coupled pair of Hodgkin-Huxley neurons.
\end{abstract}

DOI: 10.1103/PhysRevE.93.032210

\section{INTRODUCTION}

Spike timing-dependent plasticity (STDP) is an adaptive change of connections between neurons dependent on the relative timing of their action potentials. It seems to be a key mechanism involved in the processes of memory and learning by the nervous system. STDP was envisioned by Hebb already in 1949 [1], and evidence for its synaptic implementation has been found during the last decades [2-12].

For theoretical studies of neuron ensembles with adaptive coupling structure, mathematical models of different complexity have been used, which incorporate variable coupling strengths and exhibit different types of synchronization such as (cluster) coherence and incoherence [13-18]. Among those, some of the simplest models are networks of phase oscillators whose coupling strengths are subjected to plasticity. A more realistic description is obtained if more complex neuron models, e.g., the Hodgkin-Huxley model, are included as interacting elements.

Networks of phase oscillators with plasticity have been considered by several studies [13-15,18-21]. Seliger et al. [19] investigated ensembles of phase oscillators with phasedifference-dependent plasticity (PDDP) and examined the influence of plasticity parameters-in particular, the rate of the adaptive dynamics. They found parameter regions where a nonsynchronized, weakly coupled regime coexists with multiple stable clustered states with enhanced mutual connections within each cluster. Maistrenko et al. [20] studied oscillators with a different type of PDDP and showed a similar phenomenon: multistability of the desynchronized and partially synchronized cluster states. Clustering phenomena were also observed in Refs. [15,22,23].

Tass and Majtanik [13] suggested to use a desynchronizing stimulation to reliably shift a neuronal target population from a coexisting strongly coupled and synchronized regime to a weakly coupled and desynchronized regime. Such an approach can have an important impact on the treatment of neurological disorders characterized by abnormal neuronal synchronization like Parkinson's disease or tinnitus. As shown by several modeling, experimental, and clinical studies [13,24-34], pathological neuronal connectivity and synchrony can be counteracted by a desynchronizing stimulation on a longterm basis, which may lead to a significant and long-lasting amelioration of symptoms.

A recent study by Popovych et al. [18] considered a situation where ensembles of phase oscillators and spiking neurons with adaptive coupling were perturbed by an independent random input, which is known to be a powerful method for counteracting synchronization in coupled oscillators without STDP [35]. In neuronal networks with adaptive synapses, however, random noise stimulation administered to a population of strongly coupled and synchronized neurons with STDP may enhance the amount of synaptic coupling among the neurons [18]. For such a stimulation the phenomenon of self-organized resistance to noise has been reported that is characterized by an increase of the overall coupling and preservation of synchrony in the neuronal populations with STDP in response to the growth of the noise strength.

In this article, we study the mechanism underlying this phenomenon in detail and investigate the dynamics of the synaptic strengths between two coupled oscillatory neurons in the presence of noise. We first study a model where the neurons are described by phase oscillators, and natural fluctuations are modeled by Gaussian additive noise. Considering the situation, when the adaptation of the coupling strengths (plasticity) occurs on a much slower time scale than the underlying neural activity, we obtain a reduced system describing the evolution of the coupling strengths. The analysis of the reduced equation allows us to gain a deeper, to some extent analytical, insight into the role that noise, natural frequencies, 
and plasticity play for the long-term evolution of the synaptic coupling in the case of two oscillators. In particular, we find that multistability between different coupling regimes (unidirectional, bidirectional, and uncoupled) is possible. We show how the multistability is mediated by the noise intensity and frequency detuning. We provide a supporting analytical evidence to the observation in [18], that an increased level of noise can stabilize bidirectional coupling and thereby strengthen the synchronization properties of the system.

Fokker-Planck equations (FPE) for coupled phase oscillators with noise but without plasticity have been previously studied in several publications. For instance Nakao et al. [36] derived stationary solutions for an ensemble of uncoupled oscillators with common noise and Kurebayashi et al. [37] considered a similar scenario in the presence of a colored noise. Ly and Ermentrout [38] derived a phase reduction for two coupled oscillators with noise. These studies rely on FPEs for the phase differences of two oscillators, which, for the uncoupled case, allows inferences about the dynamics of a large ensemble [36,37].

In the second part of the paper, we consider a more realistic model of two interacting spiking Hodgkin-Huxley (HH) neurons, where synaptic weights are governed by STDP. The neurons are perturbed by an independent random excitatory synaptic input. For this model the update rule for the synaptic weights is based on the relative spike timing rather than the phase difference as in the case of the phase oscillator model, and the random perturbations take the form of excitatory synaptic input generated by a spike train with Gaussian distribution rather than Gaussian noise. In spite of the different update rule and the different type of random perturbations, $\mathrm{HH}$ neurons and phase oscillators demonstrate much similarity in the dynamics of coupling when parameters vary. In particular, we observed a noise-induced emergence of bidirectional coupling and a multistability of different coupling regimes in both models. These results are also in accordance with Ref. [18] which showed that the emerging coupling structure in a network of HH neurons is well resembled by the emerging structure in a network of phase oscillators.

The paper is organized as follows. Section II introduces the phase model. In Secs. III and IV we derive reduced dynamical equations for slowly changing coupling strengths. Section V is devoted to the study of the derived system for the coupling strengths. Two coupled HH neurons are considered in Secs. VI and VII, and Sec. VIII gives final conclusions.

\section{MODEL EQUATIONS}

\section{A. Phase dynamics}

As a simple model for a pair of mutually coupled neurons we consider two phase oscillators $\vartheta_{1}(t)$ and $\vartheta_{2}(t)$ with natural frequencies $\omega_{1}$ and $\omega_{2}$, coupled via a $2 \pi$-periodic coupling function $g$ and coupling weights $w_{1}$ and $w_{2}$. Further, we consider natural fluctuations in the form of independent Wiener processes $W_{1}$ and $W_{2}$ which act on the dynamics with the intensity $\sqrt{\mu}$. The corresponding stochastic differential equations [39] are given by

$$
\begin{aligned}
& d \vartheta_{1}=\left[\omega_{1}+w_{1} g\left(\vartheta_{2}-\vartheta_{1}\right)\right] d t+\sqrt{\mu} d W_{1}, \\
& d \vartheta_{2}=\left[\omega_{2}+w_{2} g\left(\vartheta_{1}-\vartheta_{2}\right)\right] d t+\sqrt{\mu} d W_{2}
\end{aligned}
$$

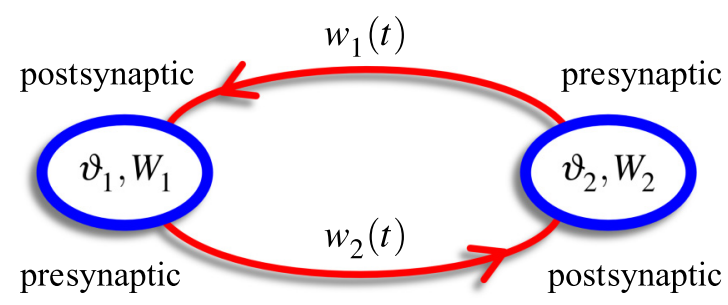

FIG. 1. Schematic representation of the model; see Eq. (1).

see Fig. 1. From the system of two coupled phase oscillators (1) we obtain the equation for the phase difference $\varphi(t)=$ $\vartheta_{2}(t)-\vartheta_{1}(t) \in[0,2 \pi)$ :

$$
d \varphi=\left[\Delta \omega+w_{2} g(-\varphi)-w_{1} g(\varphi)\right] d t+\sqrt{2 \mu} d W,
$$

with $\Delta \omega=\omega_{2}-\omega_{1}$ and $W=\left(W_{2}-W_{1}\right) / \sqrt{2}$.

In this work we are interested in the combined effects of noise and synaptic plasticity. This means the coupling weights $w_{1,2}=w_{1,2}(t)$ change in time depending on the dynamics of the phases $\vartheta_{1,2}(t)$. One common modeling assumption is that the change of $w_{1,2}(t)$ depends continuously on the phase difference; see $[15,19,20,40,41]$. This leads to dynamical equations for $w_{1,2}$, which take the form

$$
\dot{w}_{i}(t)=P_{i}[\varphi(t)],
$$

with some functions $P_{i}(\cdot)$ governing the continuous coupling update. In general these functions are much smaller in absolute value than the functions governing the dynamics of the neurons (2), since the plasticity update occurs on a slower time scale. We refer to an update of the form (3) as "phase-differencedependent plasticity" (PDDP).

Note that if both connections between $\vartheta_{1}$ and $\vartheta_{2}$ have the same type of plasticity, we have $P_{1}(\varphi)=P_{2}(-\varphi)$. Several different choices for the coupling update have been used in previous studies. For instance, Aoki et al. $[15,40]$ considered trigonometric functions of the form

$$
\dot{w}_{1}=\varepsilon \sin (\varphi-\beta),
$$

with a parameter $\beta$, which can be adjusted to mimic in a simple way plasticity rules occurring in neural connections. Maistrenko et al. [20] considered a more specific function of the form

$$
\dot{w}_{1}= \begin{cases}\left(\alpha-w_{1}\right) \exp \left(\frac{\varphi}{\tau_{p}}\right), & \varphi \in[-\pi, 0], \\ -w_{1} \exp \left(-\frac{\varphi}{\tau_{d}}\right), & \varphi \in[0, \pi] .\end{cases}
$$

Some other functions can be found in $[19,41]$. In the following Sec. II B, we consider an additive update rule based on the spike time difference and show how STDP can be related to a PDDP rule in some situations.

\section{B. PDDP versus STDP}

In the course of this work we will adopt a PDDP model, but first we comment on the relation between PDDP and STDP. In a model which describes an oscillatory neuron by its phase $\vartheta(t) \in[0,2 \pi)$, one can deliberately choose the point $\vartheta=0$ as corresponding to the moment when a spike occurs. So let us employ this convention for each neuron of the system (1). 

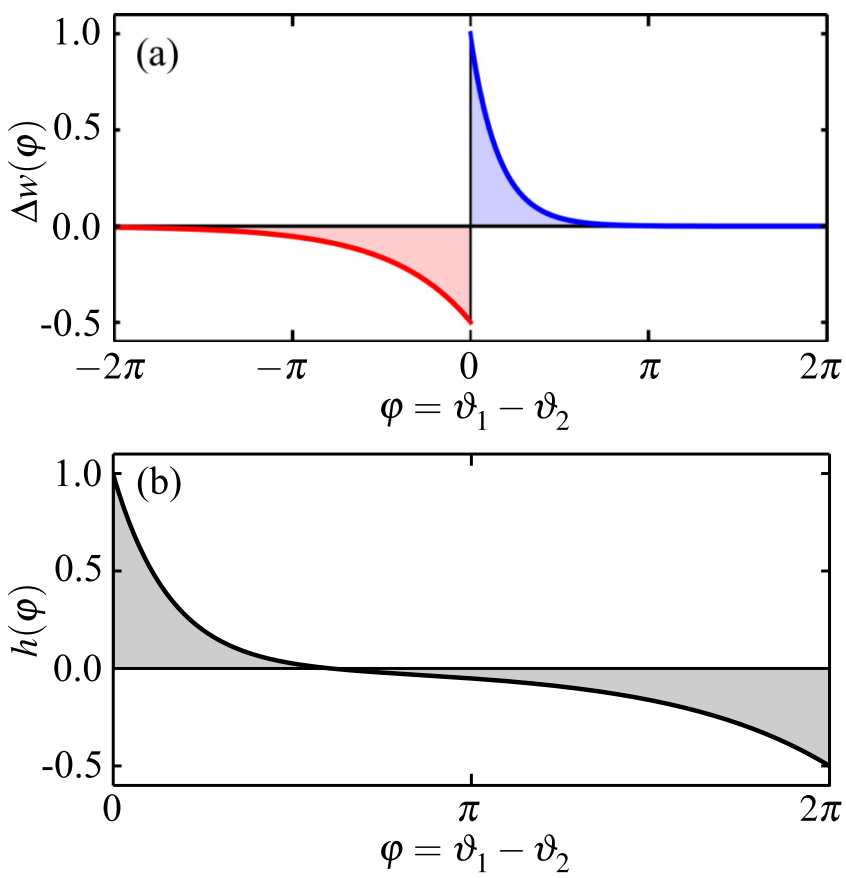

FIG. 2. (a) Plasticity function (5) with $A_{1}=1, A_{2}=0.5, \tau_{1}=$ 0.5 , and $\tau_{2}=1.4$; (b) the corresponding PDDP dynamics for $w(t) \in$ $\left(0, w_{\max }\right)$; see $(8)$.

In the case of STDP it is assumed that the coupling strength of a connection is updated each time, when one of the two connected neurons emits an action potential. Further, we assume that the STDP function depends on the time difference

$$
\Delta t=t_{\text {post }}-t_{\text {pre }}
$$

between the last spikes of both neurons. Here $t_{\text {post }}$ is the last spike time of the postsynaptic and $t_{\text {pre }}$ of the presynaptic neuron; cf. Fig. 1. We denote the value of the corresponding change in the coupling strength by $\delta \Delta w(\Delta t)$, such that $w \rightarrow w+\delta \Delta w(\Delta t)$, where $\delta$ is a parameter determining the magnitude of an individual update. In order to reflect that the coupling dynamics takes place at a much slower time scale than the underlying neuronal activity, the maximal update of synaptic weights is usually considered of order $10^{-3}$ to $10^{-4}$ of magnitude $[2,11,16]$. The approach presented in this work does not pose any special assumptions on the coupling function $g(\cdot)$ or the update function $\Delta w(\cdot)$. But wherever we have to be more specific (e.g., for illustrations) we will refer to the following form $[2,3,7,10,42]$ [see Fig. 2(a)]:

$$
\Delta w(\Delta t)= \begin{cases}\Delta w_{+}(\Delta t)=A_{1} \exp \left(-\frac{\Delta t}{\tau_{1}}\right), & \text { for } \Delta t>0, \\ \Delta w_{-}(\Delta t)=-A_{2} \exp \left(\frac{\Delta t}{\tau_{2}}\right), & \text { for } \Delta t<0, \\ 0, & \text { for } \Delta t=0\end{cases}
$$

where the plasticity parameters $A_{1}, A_{2}, \tau_{1}$, and $\tau_{2}$ are positive. In our simulations we consider the case $A_{1}>A_{2}$ and $\tau_{1}<$ $\tau_{2}$, as in Fig. 2, where potentiation dominates for small $\Delta t$, and depression dominates for large $\Delta t$, which was confirmed experimentally $[4,7,43]$.

The rule (5) implies that the synaptic strength is increased each time when the postsynaptic neuron spikes at $t_{\text {post }}$, since the last spike of the presynaptic neuron was in the past and $t_{\text {pre }}<t_{\text {post }}$, hence $\Delta t>0$. Similarly, the synaptic strength is decreased each time when the presynaptic neuron spikes, since $t_{\text {post }}<t_{\text {pre }}$ and $\Delta t<0$ at this moment. The longer the last two spike times come apart, the less is the amount of change of the coupling strength.

From a practical point of view it can be desirable to reduce the STDP rule of the phase model to a PDDP where the update occurs each time when $\vartheta_{\text {post }}=0$ or $\vartheta_{\text {pre }}=0$, and the update function depends on the phase difference $\varphi=\vartheta_{\text {pre }}-\vartheta_{\text {post }} \in$ $[0,2 \pi]$ only. The main advantage of the PDDP description is that it reduces the complexity of the model considerably.

In the case when the evolution of the phase differences is slower than the phase dynamics (e.g., if multiple spikes occur prior to a significant change of the phase difference, or if the system is close to a phase-locked state), this reduction is possible: One can approximate the STDP update $\Delta w(\Delta t)$ by an expression based on the phase difference $\varphi$. For instance, at the time when the postsynaptic neuron spikes, i.e., $\vartheta_{\text {post }}=0$, the spike time difference can be approximated as $\Delta t \approx \varphi / \Omega$, where $\Omega$ is the mean spiking frequency (e.g., frequency of the locked state). Correspondingly, the effective update is approximated by $\Delta w_{+}(\Delta t) \approx \Delta w_{+}(\varphi / \Omega)$, where we indicate the sign of the argument by a subscript as in (5). If the presynaptic neuron spikes, the (negative) spike time difference is approximately given by $\Delta t \approx(\varphi-2 \pi) / \Omega$ and $\Delta w_{-}(\Delta t) \approx \Delta w_{-}[(\varphi-2 \pi) / \Omega]$.

If the changes of coupling strengths take effect on a large time scale, that is, if the update magnitude $\delta$ is small, it is possible to approximate the discontinuous coupling strength update at spike times by a continuous change according to the update rate

$$
\dot{w}(t)=\delta \frac{\Omega}{2 \pi}\left[\Delta w_{+}\left(\frac{\varphi}{\Omega}\right)+\Delta w_{-}\left(\frac{\varphi-2 \pi}{\Omega}\right)\right] .
$$

Here $\Omega / 2 \pi$ is the number of spikes (crossings of $\vartheta_{\text {post }}=0$ ) per unit time, and the two summands correspond to the twofold update at spike times of the presynaptic and of the postsynaptic neuron. With the plasticity function (5), we obtain the update

$$
\dot{w}(t)=\frac{\delta \Omega}{2 \pi}\left[A_{1} \exp \left(-\frac{\varphi}{\Omega \tau_{1}}\right)-A_{2} \exp \left(\frac{\varphi-2 \pi}{\Omega \tau_{2}}\right)\right] .
$$

Assuming $\Omega=1$ (e.g., by rescaling time or redefining the plasticity parameters) and defining the function

$$
h(\varphi):=\frac{1}{2 \pi}\left[A_{1} \exp \left(-\frac{\varphi}{\tau_{1}}\right)-A_{2} \exp \left(\frac{\varphi-2 \pi}{\tau_{2}}\right)\right],
$$

the corresponding continuous PDDP update rule for $w_{1}(t)$ in (2) reads

$$
\dot{w}_{1}(t)=\delta h(\varphi) .
$$

In a similar way, the equation describing the dynamics of the coupling weight $w_{2}(t)$ is obtained as

$$
\dot{w}_{2}(t)=\delta h(2 \pi-\varphi) .
$$

Figures 2(a) and 2(b) compare the STDP update function (5) and the corresponding PDDP function $h(\varphi)$. One can observe the qualitative similarity of the right part of $h$ (for $\pi<\varphi<$ $2 \pi$ ) with the negative part $\Delta w_{-}$of (5), and the left part of $h$ (for $0<\varphi<\pi)$ with $\Delta w_{+}$. This is because the tails of $\Delta w(\Delta t)$ are exponentially decreasing. 
Taking into account Eqs. (9) and (10), in the following, we study the dynamics of system (2) with the PDDP rule

$$
\begin{array}{cl}
\dot{w}_{1}=\delta h(\varphi), & 0<w_{1}<w_{\max }, \\
\dot{w}_{2}=\delta h(2 \pi-\varphi), & 0<w_{2}<w_{\max } .
\end{array}
$$

To guarantee that the coupling weights remain bounded we restrict them to an interval $\left[0, w_{\max }\right]$. This is achieved modeling the natural saturation of the coupling weights via the following "hard bounds" [2,16,18,44]:

$$
\begin{aligned}
\dot{w}_{1}=\min \{\delta h(\varphi), 0\}, & \text { for } w_{1}=w_{\max }, \\
\dot{w}_{2}=\min \{\delta h(2 \pi-\varphi), 0\}, & \text { for } w_{2}=w_{\max },
\end{aligned}
$$

and

$$
\begin{aligned}
\dot{w}_{1}=\max \{\delta h(\varphi), 0\}, & \text { for } w_{1}=0, \\
\dot{w}_{2}=\max \{\delta h(2 \pi-\varphi), 0\}, & \text { for } w_{2}=0 .
\end{aligned}
$$

We note that there are also other ways of introducing the saturation, such as "soft bounds"; see Eq. (4) or Refs. [15,19,20,42,45,46].

\section{COEXISTENCE AND SWITCHING BETWEEN SYNCHRONOUS AND DESYNCHRONOUS STATES}

Systems with plasticity (STDP as well as PDDP) often exhibit multistability of desynchronized and synchronized solutions $[13,18,20,24]$. Desynchronized states are usually characterized by weak coupling strengths when the neurons behave independently from each other. Synchronized or coherent states usually appear when the coupling is strong, were the neurons show identical mean frequencies, and the phase differences are bounded. Multistability appears if several different configurations of connectivity become dynamically stable.

For instance, the uncoupled state $w_{1,2} \approx 0$ in system (2), with PDDP (9) and (10) and cut-off (12) and (13), will be stable if the coupling weights $w_{1,2}$ are in average depressed whenever they become slightly positive. Since phase differences of the uncoupled and desynchronized system are uniformly distributed, the average update is

$$
\begin{aligned}
\left\langle\dot{w}_{1}\right\rangle & =\left\langle\dot{w}_{2}\right\rangle=\frac{\delta}{2 \pi} \int_{0}^{2 \pi} h(\varphi) d \varphi \\
& =\frac{\delta}{4 \pi^{2}}\left[A_{1} \tau_{1}\left(1-e^{-2 \pi / \tau_{1}}\right)-A_{2} \tau_{2}\left(1-e^{-2 \pi / \tau_{2}}\right)\right] .
\end{aligned}
$$

In the case when this quantity is negative, there appears the following stabilizing (negative) feedback: a small intermediate increase of $w_{1}$ and $w_{2}$ causes only a small deviation of the phase distribution from the uniform one and therefore the average update still remains negative. Consequently, the coupling strengths are forced to decrease to zero again. For example, for the parameters of the plasticity function $A_{1}>A_{2}$, the uncoupled state can be stable only for $\tau_{2}>\tau_{1}$. In particular, Eq. (14) is negative for the parameters in Fig. 2, and the uncoupled state is stable.

The synchronized state of the noise-free $(\mu=0)$ system (2) with, for example, coupling function $g(\varphi)=\sin (\varphi)$ is characterized by a fixed phase difference $\varphi^{*}=\arcsin \left[\Delta \omega /\left(w_{1}+\right.\right.$
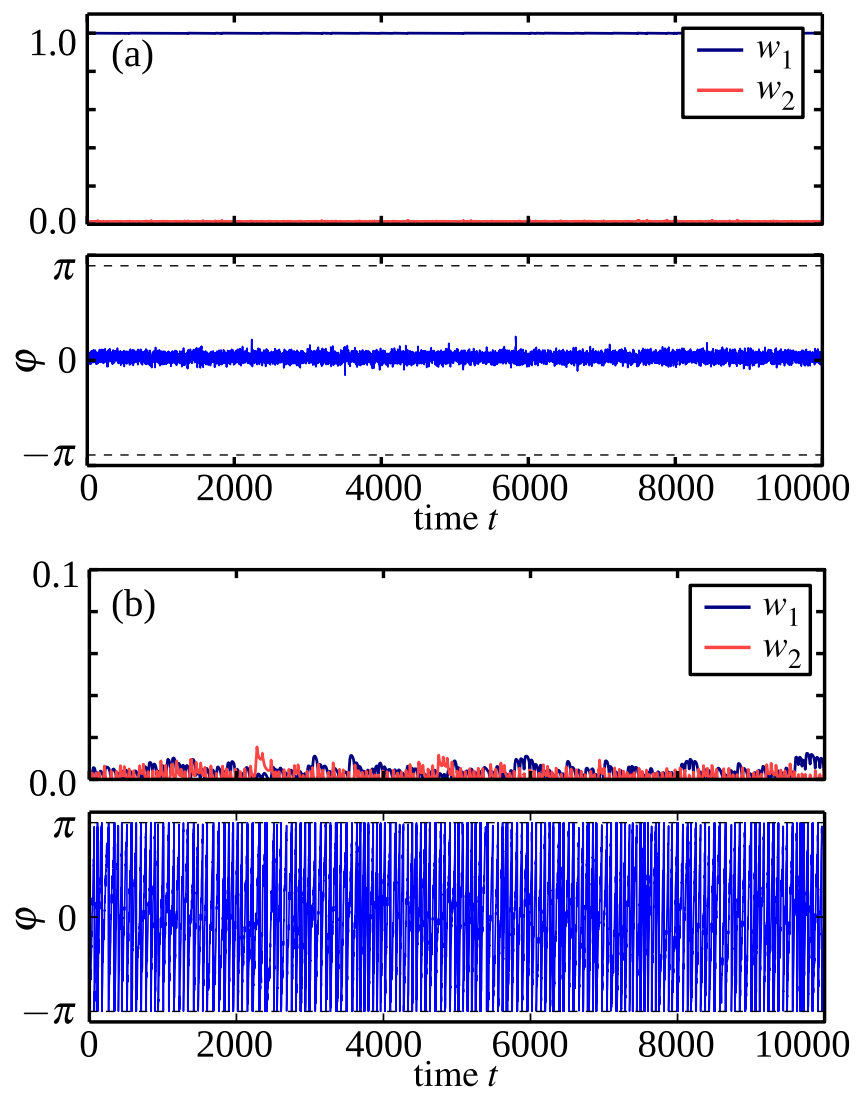

FIG. 3. Time evolution of the coupling weights and phase differences of two oscillators (2) with the coupling function $g(\varphi)=\sin (\varphi)$ and the PDDP rule (7); see Fig. 2. Plot (a) shows a unidirectionally coupled state with $w_{1} \approx w_{\max }=1$ and $w_{2} \approx 0$, and (b) shows the coexisting desynchronized (and uncoupled) state with $w_{1,2} \approx 0$. For (a) and (b) the same parameters were used: $\Delta \omega=0.1, \mu=0.01, \delta=$ 0.001 , and plasticity parameters as given in Fig. 2 .

$\left.w_{2}\right)$ ]. As follows from Eqs. (8)-(10), in this regime the coupling weight $w_{1}$ will be potentiated if

$$
\tau_{1}>\frac{\varphi^{*} \tau_{2}}{2 \pi-\varphi^{*}-\tau_{2} \ln \left(A_{2} / A_{1}\right)},
$$

and the coupling weight $w_{2}$ will be depressed if

$$
\tau_{1}<\frac{\left(2 \pi-\varphi^{*}\right) \tau_{2}}{\varphi^{*}-\tau_{2} \ln \left(A_{2} / A_{1}\right)} .
$$

In particular, the above conditions are satisfied for the parameters in Fig. 2, such that there coexist two stable coupling regimes, the uncoupled state with $\left(w_{1}, w_{2}\right) \approx(0,0)$ and unidirectional coupling $\left(w_{1}, w_{2}\right) \approx\left(w_{\max }, 0\right)$. These regimes are also preserved for small noise.

In Fig. 3 we show typical evolutions of the phase difference $\varphi(t)$ and coupling weights $w_{1}(t)$ and $w_{2}(t)$. For noise intensity $\mu=0.01$, detuning $\Delta \omega=0.1$, and sufficiently small plasticity rate $\delta=10^{-3}$, we observe two states which persist over a very long time. The first state is characterized by a unidirectional coupling $\left(w_{1}, w_{2}\right) \approx(1,0)$ and synchronization of the two neurons [Fig. 3(a)], while the second one is the uncoupled 

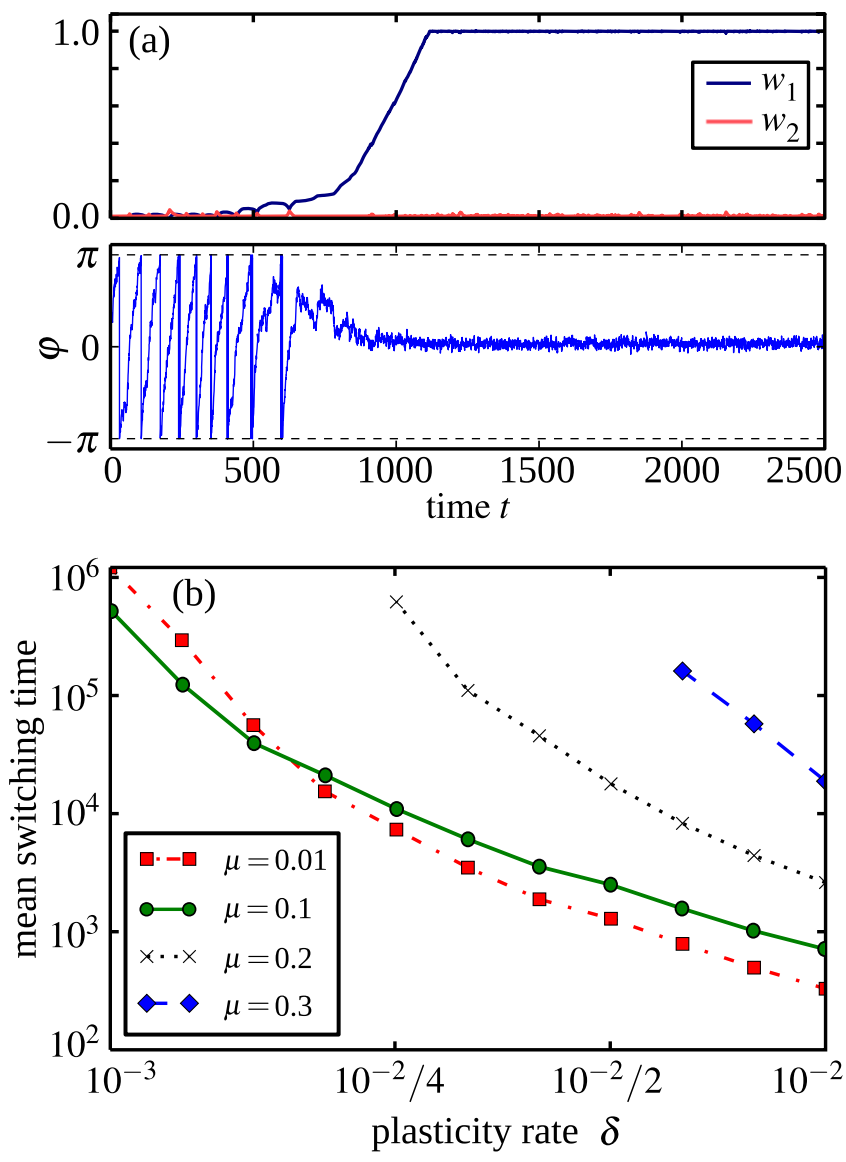

FIG. 4. Noise-induced switching between the desynchronized (uncoupled) regime and the unidirectionally coupled phase-locked regime. Plot (a) shows a single event for $\delta=0.005$ at time $t_{0} \approx 900$. Switch events were detected as the first time $t_{0}$ for which the system reaches a state with $w_{1}\left(t_{0}\right)>0.5$. In (b) the mean switching time from the uncoupled state to the unidirectionally coupled state is shown in dependence of the plasticity rate $\delta$ for $\mu=0.01,0.1,0.2$, and 0.3 . Other parameters as in Fig. 3.

and desynchronized state with $\left(w_{1}, w_{2}\right) \approx(0,0)$ [Fig. 3(b)]. Both states correspond to attractors of the deterministic system without noise $(\mu=0)$ : a stable unidirectionally coupled state with a constant phase difference $\varphi=\arcsin \left(\Delta \omega / w_{\max }\right)$ and a stable uncoupled state with small fluctuations of the coupling weights with a magnitude $\delta$.

Under the influence of noise, the system may leave the desynchronized state even if the average update (14) is negative. For the parameters as in Fig. 3 we observe switching from the uncoupled state to the unidirectionally coupled attracting state which is illustrated in Fig. 4(a). These switchings occur more often with increasing $\delta$. Figure 4(b) shows how the mean time needed until a switch occurs after the initialization with $w_{1}=w_{2}=0$ decreases with $\delta$ for $\mu=0.01,0.1,0.2$, and 0.3. In simulations we also observed that the mean switching times grow further with increased noise intensity $\mu>0.3$. Switchings from the unidirectionally coupled state back to the uncoupled state were not observed for the depicted parameter values and the considered simulation time, which may be connected to the size of the basins of attraction of these coupling regimes and their attraction rates.

\section{AVERAGED MODEL FOR THE COUPLING WEIGHTS DYNAMICS}

Under the assumption that the phase dynamics is much faster than the changes of the coupling due to plasticity (i.e., $\delta$ is small), (11) can be averaged leading to

$$
\begin{aligned}
& \dot{w}_{1}=\delta \int_{0}^{2 \pi} h(\varphi) \rho\left(t, \varphi ; w_{1}, w_{2}\right) d \varphi, \\
& \dot{w}_{2}=\delta \int_{0}^{2 \pi} h(2 \pi-\varphi) \rho\left(t, \varphi ; w_{1}, w_{2}\right) d \varphi .
\end{aligned}
$$

For the ease of notation we drop the brackets indicating averages in Secs. IV and V, i.e., $w_{j} \triangleq\left\langle w_{j}\right\rangle$. In Eq. (15) $\rho\left(t, \varphi ; w_{1}, w_{2}\right)$ is the probability density for the state of system (2) for fixed values of $w_{1}$ and $w_{2}$, and it satisfies the following Fokker-Planck equation (FPE) [39]:

$$
\partial_{t} \rho=-\partial_{\varphi}(v(\varphi) \rho)+\mu \partial_{\varphi}^{2} \rho,
$$

where

$$
v(\varphi)=\Delta \omega+w_{2} g(-\varphi)-w_{1} g(\varphi)
$$

see Eq. (2). Note that for the case of the hard bounds (12) and (13), Eqs. (15) hold within the domain $0<w_{1}<w_{\max }, 0<$ $w_{2}<w_{\max }$, and the boundaries should be treated separately.

Let us further assume that the FPE converges to some stationary distribution $\rho_{s}\left(\varphi ; w_{1}, w_{2}\right)$ on a faster time scale than the changes of coupling weights. As a result, we can further simplify (15) as follows:

$$
\begin{aligned}
& \dot{w}_{1}=\delta \int_{0}^{2 \pi} h(\varphi) \rho_{s}\left(\varphi ; w_{1}, w_{2}\right) d \varphi, \\
& \dot{w}_{2}=\delta \int_{0}^{2 \pi} h(2 \pi-\varphi) \rho_{s}\left(\varphi ; w_{1}, w_{2}\right) d \varphi .
\end{aligned}
$$

Note that for the case of the uniform distribution $\rho_{s}(\varphi ; 0,0) \equiv$ $1 / 2 \pi$, Eqs. (18) lead to the update (14). In general, they define a planar vector field with respect to $\left(w_{1}, w_{2}\right)$, describing the slow average evolution of the coupling weights.

Let us determine the stationary distribution $\rho_{s}$, which is the last unknown ingredient in Eq. (18). It must satisfy Eq. (16) with $\partial_{t} \rho_{s}=0$. By integration we obtain

$$
\partial_{\varphi} \rho_{s}(\varphi)=\frac{1}{\mu} v(\varphi) \rho_{s}(\varphi)+C
$$

with the following periodic and normalization conditions:

$$
\rho_{s}(0)=\rho_{s}(2 \pi), \quad \int_{0}^{2 \pi} \rho_{s}(\varphi) d \varphi=1 .
$$

For brevity we have omitted the dependence of $\rho_{s}$ on $w_{1}$ and $w_{2}$. Equation (19) can be solved by variation of constants formula, which yields

$$
\rho_{s}(\varphi)=e^{\int_{0}^{\varphi}(1 / \mu) v(\psi) d \psi}\left(\rho_{0}+C \int_{0}^{\varphi} e^{-\int_{0}^{\chi}(1 / \mu) v(\psi) d \psi} d \chi\right),
$$



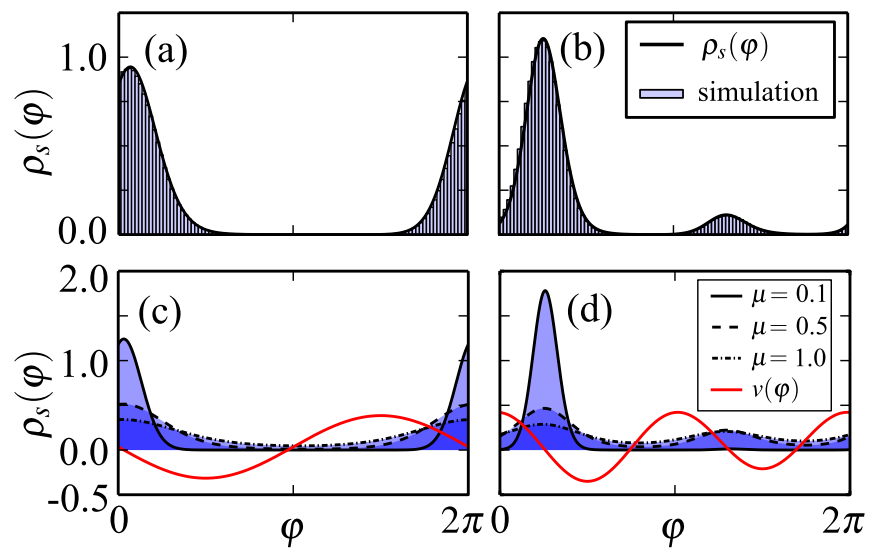

FIG. 5. Panels (a) and (b): Lines show stationary distributions $\rho_{s}(\varphi)$ given by Eq. (21) in the case of fixed unidirectional coupling $w_{1}=1, w_{2}=0$. Bars show numerically obtained histograms from the computed phase dynamics (2). The histograms consist of 100 bins, and are based upon data from a simulation over 10000 units of time. Panels (c) and (d): Stationary distributions $\rho_{s}$ for different noise intensities $\mu$ (black lines) and fixed unidirectional coupling. The red line indicates the value of the advection part $v(\varphi)$ of the phase dynamics; see (17). Panels (a) and (c): results for the coupling function $g(\varphi)=\sin (\varphi)$ and parameters $\Delta \omega=0.1, \mu=0.2$. Panels (b) and (d): $g(\varphi)=0.2 \sin (\varphi)+\cos (2 \varphi)$ and $\Delta \omega=0.2, \mu=0.2$.

where the two unknown constants $\rho_{0}=\rho_{s}(0)$ and $C$ can be determined from (20). We find

$$
C=\frac{1}{\gamma}\left(1-e^{(1 / \mu) \bar{v}}\right)
$$

where $\bar{v}=\int_{0}^{2 \pi} v(\varphi) d \varphi$,

$$
\begin{aligned}
\gamma= & \int_{0}^{2 \pi}\left(\int_{0}^{\varphi} e^{\int_{\chi}^{\varphi}(1 / \mu) v(\psi) d \psi} d \chi\right. \\
& \left.+\int_{\varphi}^{2 \pi} e^{(1 / \mu) \bar{v}-\int_{\varphi}^{\chi}(1 / \mu) v(\psi) d \psi} d \chi\right) d \varphi
\end{aligned}
$$

and

$$
\rho_{0}=\frac{1}{\gamma} \int_{0}^{2 \pi} e^{\int_{x}^{2 \pi}(1 / \mu) v(\psi) d \psi} d \chi
$$

Figure 5 shows some examples of $\rho_{s}(\varphi)$ for different parameters. It also demonstrates a good matching of $\rho_{s}(\varphi)$ with the numerically calculated histograms of $\varphi$ using (2) for fixed coupling strength.

Together with the expression (21), which determines the distribution $\rho_{s}(\varphi)$, Eq. (18) is an explicit two-dimensional system of ordinary differential equations governing the averaged update of the coupling weights. In the next section, we use the obtained system to study the influence of noise and frequency detuning on the dynamics of the coupling weights.

\section{DYNAMICS OF COUPLING WEIGHTS FOR THE CASE OF SINUSOIDAL COUPLING}

The obtained system (18)-(21) is quite general. It can be used for studying the dynamics of the coupling for arbitrary plasticity rules $h(\varphi)$ and coupling functions $g(\varphi)$. It also incorporates the effect of noise, which is included in expression (21) for $\rho_{s}(\varphi)$.

In this section, we consider the specific case when the plasticity function is of the form (8) derived from the STDP rule, and the coupling function $g(\varphi)=\sin \varphi$ as in the Kuramoto system.

Before we plot the specific bifurcation diagram, let us make some general remarks about the properties of the system (18)(21):

Property 1. For any odd or even coupling function $g(\varphi)$, the admissible region $Q=\left[0, w_{\max }\right]^{2}$ of the dynamics (18) is foliated by straight lines where the vector field takes identical values. Indeed, in this case the phase difference dynamics (2) and, hence, the function $v(\varphi)$ in Eq. (17) depends only on the sum $w_{1}+w_{2}$ [for odd $g(\varphi)$ ], respectively difference $w_{1}-w_{2}$ [for even $g(\varphi)$ ]. Consequently, the nullclines of $w_{1}$ and $w_{2}$, i.e., the sets with $\dot{w}_{1}=0$ and $\dot{w}_{1}=0$, consist of diagonals where the value of $w_{1}+w_{2}$ (respectively $w_{1}-w_{2}$ ) is constant and segments of the boundary of the domain $Q$. As a result, if a fixed point $\left(w_{1}^{*}, w_{2}^{*}\right)$ occurs within the interior of the domain $Q$, it leads to a line of fixed points corresponding to $w_{1}+w_{2}=$ $w_{1}^{*}+w_{2}^{*}$ (or $w_{1}-w_{2}=w_{1}^{*}-w_{2}^{*}$, respectively). For a coupling function $g$, which is neither odd nor even, isolated fixed points, stable and unstable, can also occur within $Q$.

Property 2. For the considered plasticity function (8) and the coupling $g(\varphi)=\sin \varphi$ we observe numerically that $\dot{w}_{1}>\dot{w}_{2}$ holds for $\Delta \omega>0$. As a result, we did not observe continua of fixed points which may in principle appear when two diagonal nullclines coincide. Furthermore, maximally one diagonal nullcline exists for each coupling strength and the uncoupled state $w_{1}=w_{2}=0$ is attracting, i.e., $(14)<0$. As a consequence, all stable fixed points $\left(w_{1}^{*}, w_{2}^{*}\right)$ are contained in the corners of $Q$; see Figs. 6(a)-6(f). Note that neither the noise intensity $\mu$ nor the frequency detuning $\Delta \omega$ can change the type of stability of the uncoupled state in (18).

Property 3. For an odd coupling function it is not possible that both unidirectionally coupled states are attracting, since the vector field takes the same values in the vicinity of the points $\left(w_{1}, w_{2}\right)=\left(w_{\max }, 0\right)$ and $\left(w_{1}, w_{2}\right)=\left(0, w_{\max }\right)$ and therefore the stability of one unidirectionally coupled state implies the instability of its counterpart. However, it is possible that, besides the uncoupled state, either one unidirectionally coupled state or the bidirectionally coupled state or both of them are stable at the same time [Figs. 6(a)-6(f)].

Figure $6(\mathrm{~g})$ shows a bifurcation diagram in the $(\mu, \Delta \omega)$ plane for system (18) with $g(\varphi)=\sin \varphi$. The stability regions of the bidirectionally coupled state $w_{1}=w_{2}=w_{\max }$ and the unidirectionally coupled state $\left(w_{1}, w_{2}\right)=\left(w_{\max }, 0\right)$ are indicated. The letters (a)-(f) refer to the different cases for the flow as depicted in the corresponding panels. The parameter region where the bidirectionally coupled state is stable is shaded in blue, and the stability regions of the unidirectionally coupled state is shaded in red. For each state there exist two bifurcation lines, which can bound the stability region. Each line denotes a set of parameters where one of the components of the vector field, $\dot{w}_{1}$ or $\dot{w}_{2}$, changes its sign. Consider, for example, $\Delta \omega=0.1$ and $\mu=2.5$ [region (f)], then the bidirectionally coupled state is unstable since $\dot{w}_{1}<0$ and $\dot{w}_{2}<0$, that is, both coupling weights decrease. If we decrease the noise intensity to $\mu=1.5$ [region (c)] both blue 

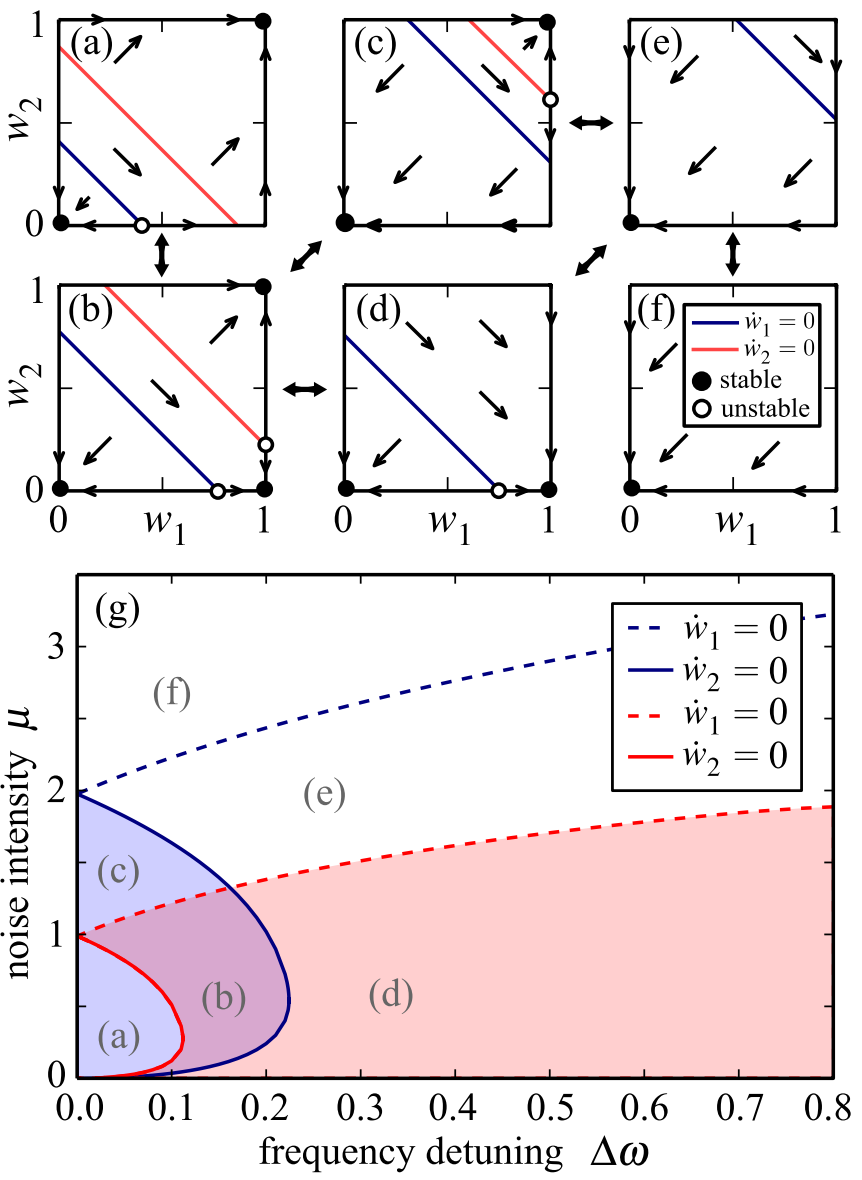

FIG. 6. Phase portraits and bifurcation diagram for system (18) with $g(\varphi)=\sin \varphi$ and $h(\varphi)$ as in Fig. 2. Panels (a), (c): uncoupled and bidirectionally coupled states are stable; (b) uncoupled, unidirectionally and bidirectionally coupled states are stable; (d) uncoupled and unidirectionally coupled states are stable; (e), (f) only the uncoupled state is stable. A bold arrow between two phase portraits indicates that these regimes are connected by a bifurcation [solid lines in $(\mathrm{g})$ ]. Panel $(\mathrm{g})$ shows the corresponding bifurcation diagram in the $(\Delta \omega, \mu)$ plane. Blue-shaded region: parameters, for which the bidirectional coupling $\left(w_{1}=w_{2}=w_{\max }=1\right)$ is stable [cf. (a)-(c)]. Red-shaded region: parameter for which the unidirectional coupling $\left(w_{1}=w_{\max }=1, w_{2}=w_{\min }=0\right)$ is stable [cf. (b) and (d)]. For all parameters $\Delta \omega \neq 0$ and $\mu$, the uncoupled state $w_{1}=w_{2}=0$ is stable.

lines are crossed which indicate that $\dot{w}_{1}$ and $\dot{w}_{2}$ changed their sign at the bidirectionally coupled state. Hence, at $\mu=1.5$ and close to the bidirectionally coupled state, the direction of the flow is now $\dot{w}_{1}>0$ and $\dot{w}_{2}>0$, and it points towards $\left(w_{\max }, w_{\max }\right)$. Since the coupling weights are bounded by $w_{\max }$ they get "trapped" in the corner $w_{1}=w_{2}=w_{\max }$. The shaded blue region summarizes all parameter values where bidirectional coupling is stable [regions (a)-(c)]. Similarly, the unidirectionally coupled state $w_{1}=w_{\max }, w_{2}=0$, is stable if $\dot{w}_{1}>0$ and $\dot{w}_{2}<0$, and the system can be attracted to the corresponding corner of $Q$. The parameter region where this is the case is shaded in red [regions (b) and (d)]. As mentioned above, in addition to the considered states, the uncoupled state with $w_{1}=w_{2}=0$ is stable independently on $\mu$ and $\Delta \omega$.
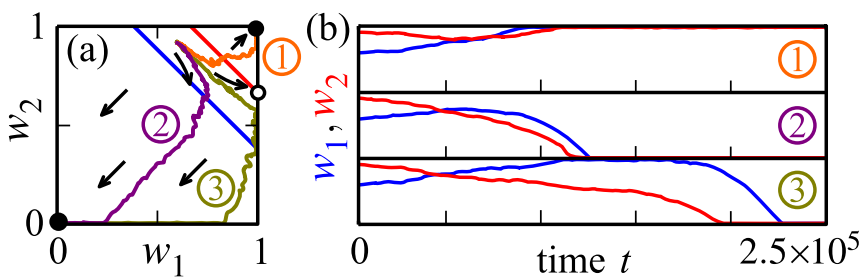

FIG. 7. Three realizations of the stochastic system (1) with (8)(10) starting from the same initial condition for the coupling weights $\left(w_{1}(0), w_{2}(0)\right)=(0.6,0.92)$ and converging either to a bidirectional coupling (trajectory 1 ) or to unidirectional coupling (trajectories 2 and 3). Parameters $\Delta \omega=0.05$ and $\mu=1.5$, which correspond to the case (c) in Fig. 6.

One may notice that the bifurcation curves of unidirectional and bidirectional coupling in Fig. 6(g) are rescaled copies of each other. This is due to the odd symmetry in $g(\varphi)$ and a parameter redundancy in (16). In fact, the rescaling

$$
\left(w_{1}, w_{2}\right) \mapsto\left(c w_{1}, c w_{2}\right), c>0,
$$

of the coupling weights is equivalent to the simultaneous rescaling of time, frequency detuning, and noise intensity as

$$
(t, \Delta \omega, \mu) \mapsto\left(c t, \frac{\Delta \omega}{c}, \frac{\mu}{c}\right) .
$$

This means that a weakly coupled pair of neurons may be equally conceived as a strongly coupled pair with large detuning and high noise intensity.

For the case of odd coupling $g(\varphi)$, Eqs. (23) and (24) imply that the value of the vector field $\left(\dot{w}_{1}, \dot{w}_{2}\right)$ at $\left(w_{1}, w_{2}\right)=(1,1)$ and $(\mu, \Delta \omega)=\left(\mu_{0}, \Delta \omega_{0}\right)$ coincides with its value at $\left(w_{1}, w_{2}\right)=$ $(1,0)$ and $(\mu, \Delta \omega)=\left(\mu_{0} / 2, \Delta \omega_{0} / 2\right)$. Therefore, the curves $\dot{w}_{1,2}=0$ for $\left(w_{1}, w_{2}\right)=(1,0)$ (red line color) are rescaled by a factor 0.5 in comparison to the corresponding curves for $\left(w_{1}, w_{2}\right)=(1,1)$ (blue line color).

Figure 7 illustrates three trajectories of the original stochastic system (1) starting at the point $\left(w_{1}(0), w_{2}(0)\right)=(0.6,0.92)$, where $\dot{w}_{1}=-\dot{w}_{2}$. This point lies close to a separatrix in the system (18), which bounds the basins of the uncoupled and the bidirectionally coupled state. It is the stable manifold of the unstable fixed point at the intersection of $\left\{\dot{w}_{2}=0\right\}$ with the boundary of the phase space $Q$. For trajectories of the stochastic system which start near this separatrix it is possible to end in both attractors either via a rather direct transition or via a longer transition which spends some time near the seperatrix and the unstable fixed point. The three shown realizations illustrate these possible behaviors.

The most remarkable observation from the diagram in Fig. 6(g) is that it shows how an increasing noise intensity may lead to stabilization of a more strongly coupled state. Thereby the noise indirectly enhances the synchronization properties of the system which is somewhat counterintuitive and can be referred to as a self-organized noise resistance [18].

The simultaneous stability of the uncoupled and the unidirectionally coupled regimes, which can be observed for vanishing noise intensity $\mu$ and finite frequency detuning $\Delta \omega$, generalizes to the case of hierarchical coupling which was 
found to be stable for ensembles of many coupled neurons as well $[18,20]$.

\section{TWO HODGKIN-HUXLEY NEURONS}

In this section we consider a system of two interacting Hodgkin-Huxley $(\mathrm{HH})$ neurons $[47,48]$,

$$
\begin{aligned}
C \dot{V}_{1,2}= & I_{1,2}-g_{N a} m_{1,2}^{3} h_{1,2}\left(V_{1,2}-V_{N a}\right) \\
& -g_{K} n_{1,2}^{4}\left(V_{1,2}-V_{K}\right)-g_{l}\left(V_{1,2}-V_{l}\right) \\
& +0.5\left(V_{r}-V_{1,2}\right) w_{1,2} s_{2,1}+I_{1,2}^{\text {input }}, \\
\dot{m}_{1,2}= & \alpha_{m}\left(V_{1,2}\right)\left(1-m_{1,2}\right)-\beta_{m}\left(V_{1,2}\right) m_{1,2}, \\
\dot{h}_{1,2}= & \alpha_{h}\left(V_{1,2}\right)\left(1-h_{1,2}\right)-\beta_{h}\left(V_{1,2}\right) h_{1,2}, \\
\dot{n}_{1,2}= & \alpha_{n}\left(V_{1,2}\right)\left(1-n_{1,2}\right)-\beta_{n}\left(V_{1,2}\right) n_{1,2}, \\
\dot{s}_{1,2}= & \frac{0.5\left(1-s_{1,2}\right)}{1+\exp \left[-\left(V_{1,2}+5\right) / 12\right]}-2 s_{1,2},
\end{aligned}
$$

where the variables $V_{1,2}$ model the membrane potentials of the neurons, and $\alpha_{m}(V)=(0.1 V+4) /[1-\exp (-0.1 V-4)]$, $\beta_{m}(V)=4 \exp [(-V-65) / 18], \quad \alpha_{h}(V)=0.07 \exp [(-V-$ $65) / 20], \beta_{h}(V)=1 /[1+\exp (-0.1 V-3.5)], \alpha_{n}(V)=$ $(0.01 V+0.55) /[1-\exp (-0.1 V-5.5)]$, and $\beta_{n}(V)=0.125$ $\exp [(-V-65) / 80]$. The neurons are excitatorily coupled (with reversal potential $V_{r}=20 \mathrm{mV}$; the results reported below are qualitatively preserved also for $V_{r}=0 \mathrm{mV}$ ) via chemical synapses with synaptic weights $w_{1,2}$ modeling the strength of the coupling from the presynaptic neuron to the postsynaptic neuron; see Fig. 1. The constant currents $I_{1}=I-\Delta I$ and $I_{2}=I+\Delta I$ with $I=11 \mu \mathrm{A} / \mathrm{cm}^{2}$ control the spiking frequencies $f_{1,2}$ (number of spikes per second) of the neurons which periodically fire with, for example, $f_{1,2} \approx 70.71 \mathrm{~Hz}$ for $\Delta I=0 \mu \mathrm{A} / \mathrm{cm}^{2}$, and $f_{1} \approx 70.44$ $\mathrm{Hz}$ and $f_{2} \approx 70.99 \mathrm{~Hz}$ for $\Delta I=0.12 \mu \mathrm{A} / \mathrm{cm}^{2}$. Other parameters $C=1 \mu \mathrm{F} / \mathrm{cm}^{2}, V_{N a}=50 \mathrm{mV}, V_{K}=-77 \mathrm{mV}$, $V_{l}=-54.4 \mathrm{mV}, g_{N a}=120 \mathrm{mS} / \mathrm{cm}^{2}, g_{K}=36 \mathrm{mS} / \mathrm{cm}^{2}$, and $g_{l}=0.3 \mathrm{mS} / \mathrm{cm}^{2}$.

The excitatory synaptic input current $I_{i}^{\text {input }}, i=1,2$, reads

$$
I_{i}^{\text {input }}(t)=\mu\left[V_{r}-V_{i}(t)\right] \sum_{\tau_{i, k}<t} \alpha\left(t-\tau_{i, k}\right) e^{-\alpha\left(t-\tau_{i, k}\right)} .
$$

The $\alpha$ train in Eq. (26) models the synaptic activity reflecting a pulsatile synaptic input received by neuron $i$ at times $\tau_{i, k}$ with the length of interpulse intervals $\Delta \tau_{i, k}=\tau_{i, k+1}-\tau_{i, k} \geqslant$ 0 independently drawn from a Gaussian distribution with mean $\left\langle\Delta \tau_{i, k}\right\rangle=14 \mathrm{~ms}$ and standard deviation $4 \mathrm{~ms}$, and $\alpha=24 /\left\langle\Delta \tau_{i, k}\right\rangle$. The mean time scale of such an input approximately equals the mean period of the coupling- and input-free neurons (25), and each neuron receives an independent random synaptic input of intensity $\mu$, which does not significantly perturb its natural spiking frequency.

The neural ensemble (25) is equipped with STDP, where the synaptic weights $w_{i}=w_{i}(t)$ are functions of time and updated as $w_{i} \rightarrow w_{i}+\delta \Delta w(\Delta t)$ according to the STDP function (5) with parameters $A_{1}=1, A_{2}=0.5, \tau_{1}=1.8 \mathrm{~ms}, \tau_{2}=6 \mathrm{~ms}$, and $\delta=0.0005$. $\Delta t$ is the time difference between the nearest spike onsets of the neurons. The spike onsets are detected by the upward zero crossing by the membrane potential following an interspike hyperpolarization. The synaptic weights $w_{i}$ are
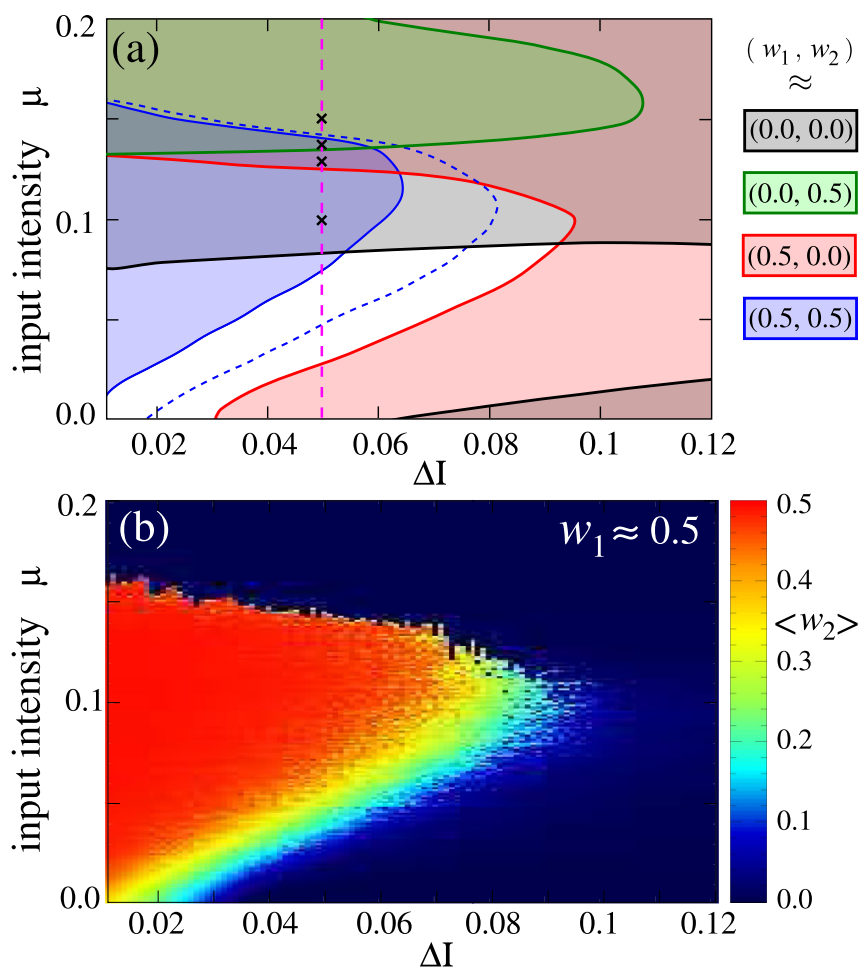

FIG. 8. Noise-induced coupling regimes of two interacting $\mathrm{HH}$ neurons (25) with random input vs parameters $\Delta I$ and $\mu$. (a) The shaded domains correspond to parameter regions where different coupling configurations are stable. The stability region of the unidirectional coupling $\left(w_{1}, w_{2}\right) \approx(0.5,0.0)$ is shaded in red, and the bidirectional coupling $\left(w_{1}, w_{2}\right) \approx(0.5,0.5)$ in blue as in Fig. $6(\mathrm{~g})$. The uncoupled state $\left(w_{1}, w_{2}\right) \approx(0.0,0.0)$ is stable in the gray shaded region, and the stability region of the inverse unidirectional coupling $\left(w_{1}, w_{2}\right) \approx(0.0,0.5)$ is indicated by green shading. The level curve $\left\langle w_{2}\right\rangle=0.25$ with $w_{1} \approx 0.5$ is depicted by blue dashed curve. The vertical magenta dashed line indicates a cross section of the parameter plane used in Figs. 9 and 10. (b) The values of the time-averaged synaptic weight $\left\langle w_{2}\right\rangle$ observed in a stable configurations with $w_{1} \approx$ 0.5 are encoded in color.

confined to the interval $\left[w_{\min }, w_{\max }\right]=[0,0.5]$ by setting $w_{i}$ to $w_{\min }$ as soon as it is depressed to a lower value than $w_{\min }$ via STDP or, respectively, to $w_{\max }$ if it is potentiated over this value. As for the phase oscillators, we consider the additive update rule for synaptic weights with hard bounds $[2,16,18,44]$. In the Appendix we also illustrate the impact of noise and parameter mismatch on the connectivity for the multiplicative update rule with soft bounds; see also Refs. [18,45].

To illustrate the noise-induced dynamics of the synaptic weights for the $\mathrm{HH}$ neurons (25), we scan the parameter space $(\Delta I, \mu)$ for possible regimes and summarize the results in Fig. 8. To numerically address the stability of the above coupling regimes we simulate the $\mathrm{HH}$ neurons (25) during $2000 \mathrm{~s}$ [the time in Eq. (25) is in ms] and check whether the trajectory escapes from a given coupling regime. In such a way the stability regions were obtained, which are illustrated in Fig. 8(a) with smoothed boundaries. We found that following coupling configurations can be stabilized by the random input: 
Full bidirectional coupling $\left(w_{1}, w_{2}\right) \approx(0.5,0.5)$. This regime can be observed for parameters from the blue shaded domain in Fig. 8(a).

Unidirectional coupling $\left(w_{1}, w_{2}\right) \approx(0.5,0.0)$. This regime can be observed for parameters from the red shaded domain in Fig. 8(a). In this regime the fast neuron 2 drives the slow neuron 1 , and coupling in the opposite direction vanishes. Such a coupling configuration can also be stable for the input-free $(\mu=0)$ system $(25)$.

Inverse unidirectional coupling $\left(w_{1}, w_{2}\right) \approx(0.0,0.5)$. This regime can be observed for parameters from the green shaded domain in Fig. 8(a), containing parameter values with large random perturbations and moderate frequency detuning.

Uncoupled state $\left(w_{1}, w_{2}\right) \approx(0.0,0.0)$. This regime can be observed for parameters from the gray shaded domain in Fig. 8(a), i.e., for sufficiently large random perturbations but as well for small random perturbations if the detuning $\Delta I$ is sufficiently large. Such a coupling configuration can also be stable for the input-free $(\mu=0)$ system $(25)$.

Note that there exists a white region in Fig. 8(a), where none of the above states can be found. This is because in this region the observed mean value $\left\langle w_{2}\right\rangle$ takes intermediate values between $w_{\min }=0.0$ and $w_{\max }=0.5$. Its continuous dependence on the parameters corresponds to the continuous color gradient in Fig. 8(b), where the value of $\left\langle w_{2}\right\rangle$ is encoded in color for coupling configurations with $w_{1} \approx 0.5$. In the red region of Fig. 8(b) $\left\langle w_{2}\right\rangle$ reaches its maximal value, i.e., $\left\langle w_{2}\right\rangle \approx 0.5$. This domain corresponds to the bidirectional coupling $\left(w_{1}, w_{2}\right) \approx(0.5,0.5)$ [blue shaded domain in Fig. 8(a)], whereas the region where $\left\langle w_{2}\right\rangle$ approaches its minimal value $\left\langle w_{2}\right\rangle \approx 0.0$ corresponds to the unidirectional coupling $\left(w_{1}, w_{2}\right) \approx(0.5,0.0)$ [red shaded domain in Fig. 8(a)]. In Sec. VII B we provide a more detailed illustration of this phenomenon, which is not observed for the phase oscillators with sinusoidal coupling considered in Sec. V.

As discussed for the phase oscillators, the random input may force the system to escape from a stable state and to switch to a different coupling regime; see Fig. 4. For the above coupling regimes we also performed a more detailed analysis (see below) for a fixed value of the parameter detuning $\Delta I=$ 0.05 indicated by the vertical magenta dashed line in Fig. 8(a).

As follows from Fig. 8 , the uncoupled state $\left(w_{1}, w_{2}\right) \approx(0,0)$ is unstable if the input intensity $\mu<0.082$ for $\Delta I=0.05$ [i.e., in the complement of the gray domain in Fig. 8(a)]. For such parameter values the trajectory escapes from the uncoupled state and switches to another existing stable coupling regime, see Fig. 8, in a relatively short time. We calculate such a mean switching time by averaging over 200 trajectories initiated at the uncoupled state and plot it versus $\mu$ in Fig. 9(a). For example, for $\Delta I=0.05$ and $\mu=0$ the system escapes from the uncoupled regime and switches to the unidirectional coupling in $27 \mathrm{~s}$ in average [Fig. 9(a)]. The mean switching time increases by several orders of magnitude as parameter $\mu$ leaves the instability interval and quickly reaches the selected maximal simulation time of $300000 \mathrm{~s}$ [Fig. 9(a)]. Therefore, for parameter values within the gray domain in Fig. 8(a) the HH neurons (25) can exhibit the uncoupled regime for a very long time.

The inverse unidirectional coupling $\left(w_{1}, w_{2}\right) \approx(0.0,0.5)$ is apparently unstable in the parameter domain complementary
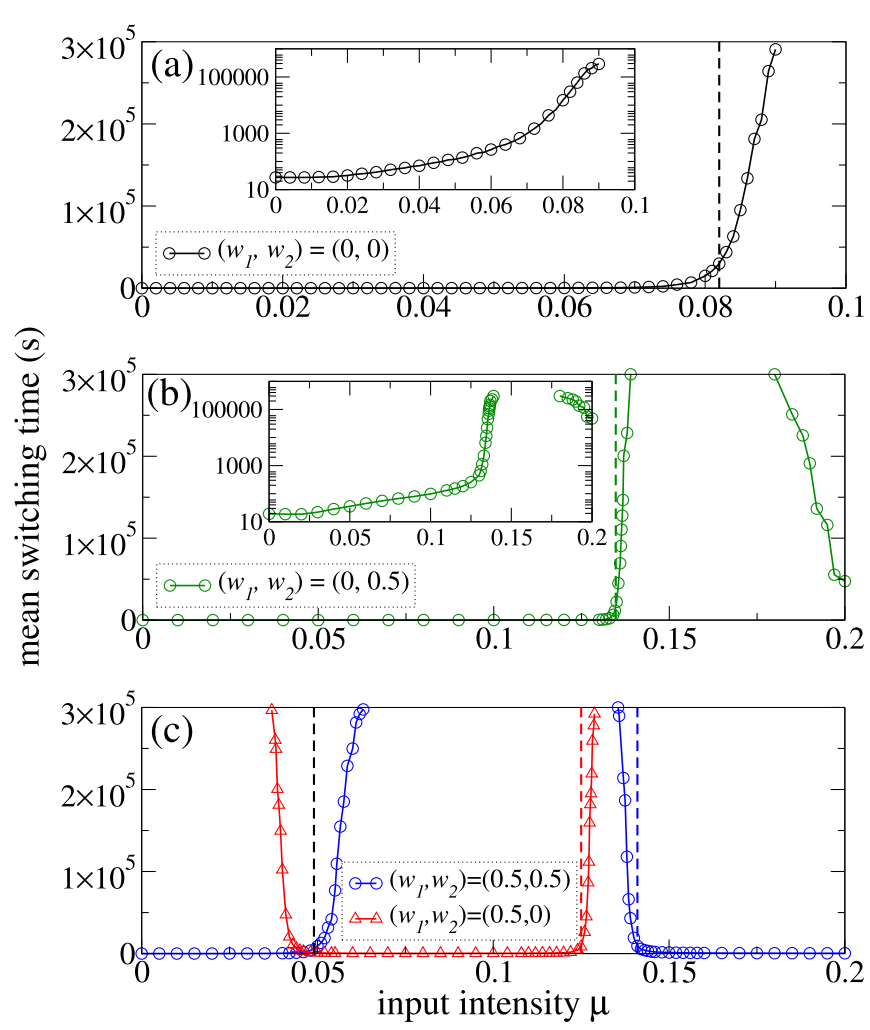

FIG. 9. Mean switching time of (a) uncoupled state, (b) inverse unidirectional coupling, and (c) bidirectional and unidirectional coupling vs input intensity $\mu$ for the fixed parameter of detuning $\Delta I=0.05$ indicated by the magenta dashed line in Fig. 8(a). The vertical dashed lines in the plots indicate the border values of $\mu$ of stability domains [i.e., the intersection of the vertical magenta dashed line in Fig. 8(a) with the borders of the corresponding stability regions]. In plot (c) the value $\mu=0.049$ of the intersection of the vertical magenta dashed line $\Delta I=0.05$ with the level curve $\left\langle w_{2}\right\rangle=0.25$ in Fig. 8(a) is indicated by black dashed line instead. The insets show plots in linear-log scale. The mean switching time was obtained by averaging over 200 trajectories initiated at the corresponding coupling regime. The maximal simulation time was $300000 \mathrm{~s}$.

to the green shaded region in Fig. 8(a), where the trajectory can relatively quickly escape from this regime. The mean switching time from the inverse unidirectional coupling is shown in Fig. 9(b) versus $\mu$ for $\Delta I=0.05$. On the other hand, for parameter values from the stability region [Fig. 8(a), green shaded region], the mean switching time gets significantly larger [Fig. 9(b)] such that the HH neurons exhibit this coupling regime for a long time in spite of relatively strong random perturbations.

The mean switching time for the bidirectional coupling $\left(w_{1}, w_{2}\right) \approx(0.5,0.5)$ and the unidirectional coupling $\left(w_{1}, w_{2}\right) \approx(0.5,0.0)$ demonstrates similar rapid growth and exceeds the selected maximal simulation time [Fig. 9(c)] as parameters enter the stability regions depicted by blue and red shaded domains, respectively, in Fig. 8(a). As mentioned above, for small values of the input intensity $\mu$, there is a gradual transition between these coupling regimes where the mean synaptic weight $\left\langle w_{2}\right\rangle$ can attain intermediate values 
between $w_{\min }=0.0$ and $w_{\max }=0.5$; see Figs. 8 (a) (white region) and 8(b). We therefore indicate the intersection value $\mu=0.049$ of the line $\Delta I=0.05$ [Figs. 8(a), vertical magenta dashed line] with the intermediate level curve $\left\langle w_{2}\right\rangle=0.25$ by a vertical black dashed line in Fig. 9(c).

\section{DIVERSITY OF STATES IN HH NEURONS}

In the following we highlight two aspects of the $\mathrm{HH}$ dynamics which were not observed for phase oscillators with sinusoidal coupling as studied in Sec. V. First we take a closer look at the multistability in the $\mathrm{HH}$ dynamics and subsequently we explain the presence of a stable configuration where $w_{2}$ takes intermediate values between $w_{\min }=0$ and $w_{\max }=0.5$.

\section{A. Multistability}

The noise-induced coupling regimes can coexist with each other, and the $\mathrm{HH}$ neurons converge to a particular state depending on the initial conditions. By varying the initial synaptic weights $w_{1}(0)$ and $w_{2}(0)$ we calculate the basins of attractions of the above coupling regimes and plot them in Fig. 10 for a few values of parameter $\mu$ and fixed $\Delta I=0.05$ indicated by crosses on the vertical magenta dashed line in Fig. 8(a). As follows from Figs. 8(a) and 10, the four coupling regimes may coexist in different combinations:

(i) Bistable regime, where uncoupled state $\left(w_{1}, w_{2}\right) \approx$ $(0,0)$ coexists with bidirectional coupling $\left(w_{1}, w_{2}\right) \approx(0.5,0.5)$ [Fig. 10(a)] or with unidirectional coupling $\left(w_{1}, w_{2}\right) \approx(0.5,0)$ (not shown).

(ii) Three coexisting coupling regimes, where uncoupled state and unidirectional coupling coexist with bidirectional coupling [Fig. 10(b)] or with inverse unidirectional coupling $\left(w_{1}, w_{2}\right) \approx(0,0.5)$ [Fig. 10(d)].

(iii) All four coupling regimes may coexist [Fig. 10(c)].

Since the neurons (25) are perturbed by an independent random input, the system may converge to different coupling regimes even for a fixed initial synaptic weight $\left(w_{1}(0), w_{2}(0)\right)$, in particular, for those close to the boundaries of the basins of attraction [Fig. 10]. This was reported for the phase oscillators; see Fig. 6 (lower panel). To illustrate such a property for $\mathrm{HH}$ neurons, we consider parameters $(\Delta I, \mu)=(0.05,0.137)$, where all four coupling regimes coexist [Figs. 8(a) and 10(c)], and simulate system (25) for fixed initial synaptic weights $\left(w_{1}(0), w_{2}(0)\right)=(0.17,0.4)$ [Fig. $10(\mathrm{c})$, black dot] and 31 different random initial conditions for neurons' variables. We found that 5 such trajectories were attracted to the bidirectionally coupled regime [Fig. 10(e)], 14 to unidirectional coupling [Fig. 10(f)], 4 to inverse unidirectional coupling [Fig. 10(g)], and 8 to the uncoupled state [Fig. 10(h)]. Since most of the trajectories converged to the unidirectional coupling, the considered initial synaptic weight has been assigned to the basin of attraction of this coupling regime [Fig. 10(c)]. The probability of being attracted to the corresponding coupling regime grows for parameters from the interior of the basins of attraction shown in Fig. 10. For example, all 31 trajectories are attracted to the bidirectional coupling for $\left(w_{1}(0), w_{2}(0)\right)=(0.45,0.4)$, to unidirectional coupling for $\left(w_{1}(0), w_{2}(0)\right)=(0.45,0.1)$, to inverse unidirectional coupling for $\left(w_{1}(0), w_{2}(0)\right)=(0.05,0.4)$, and to the uncoupled state
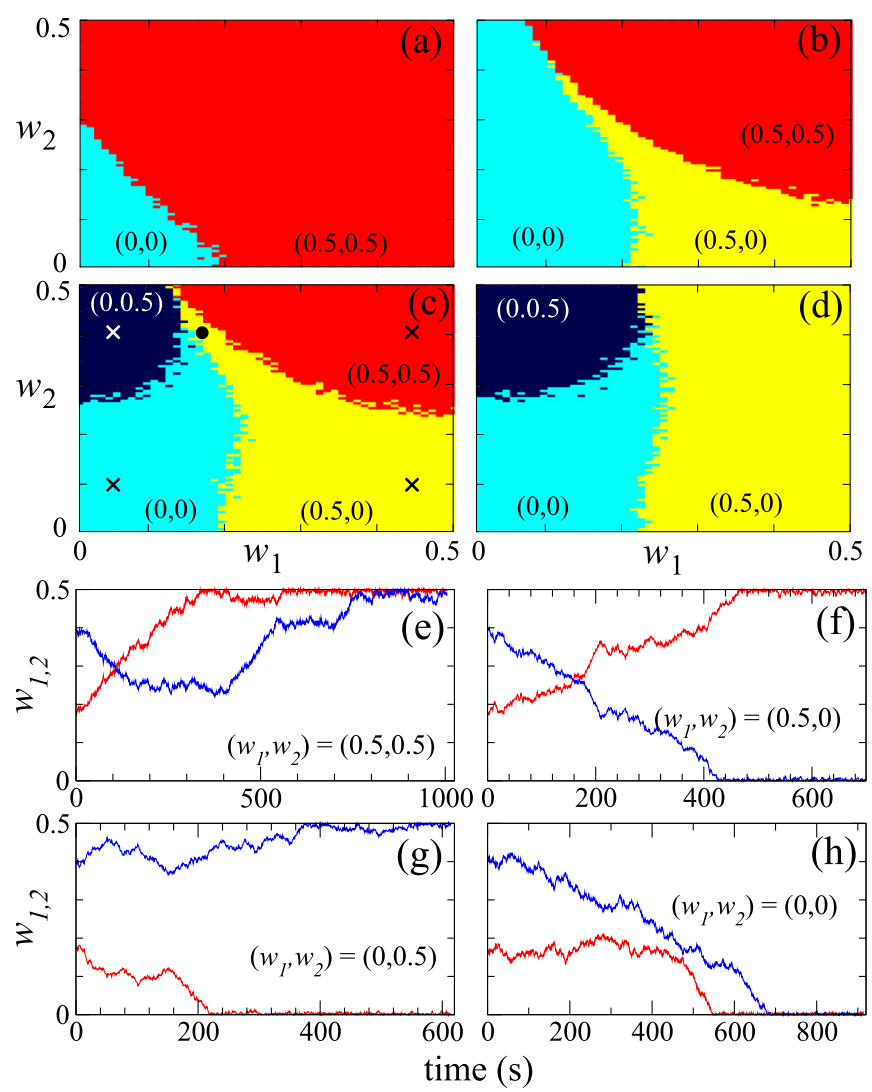

FIG. 10. (a)-(d) Basins of attraction of coexisting coupling regimes of $\mathrm{HH}$ neurons (25) depicted by color in the plane of the initial synaptic weights $\left(w_{1}(0), w_{2}(0)\right)$ for (a) $\mu=0.1$, (b) $\mu=0.132$, (c) $\mu=0.137$, and (d) $\mu=0.15$ and fixed $\Delta I=0.05$; see Fig. 8(a) (markers on the vertical magenta dashed line). The corresponding coupling regimes are indicated in the plots. Black dot and crosses in (c) indicate initial synaptic weights used for (e)-(h). Any fixed initial synaptic weight was assigned to the basin of attraction of that coupling regime to which the most of 31 different trajectories have been attracted after skipped transient. (e)-(h) Examples of the time courses of synaptic weights $w_{1}(t)$ (red curves) and $w_{2}(t)$ (blue curves) in system (25) for $\mu=0.137$ [as in (c)], fixed initial synaptic weights $\left(w_{1}(0), w_{2}(0)\right)=(0.17,0.4)$ [black dot in (c)], and random initial conditions for neurons' variables. The coupling regimes reached by the neurons after transient are indicated in the plots.

for $\left(w_{1}(0), w_{2}(0)\right)=(0.05,0.1)$; see Fig. 10(c), where the mentioned initial synaptic weights are indicated by crosses.

Note that in the case of coupled phase oscillators with sinusoidal coupling as considered in Sec. $\mathrm{V}$ the inverse unidirectional state $\left(w_{1}, w_{2}\right)=\left(0, w_{\max }\right)$ was not observed at all. In fact, the simultaneous stability of all four coupling configurations with $w_{1,2} \in\left\{w_{\min }, w_{\max }\right\}$ is not possible for odd coupling functions like $\sin (\varphi)$ since the vector field takes identical values along the diagonals $w_{1}-w_{2} \equiv$ const [cf. Fig. 6 and property 3 in Sec. V].

\section{B. Intermediate values of the coupling strength $w_{2}$}

Another feature which is present for coupled HH neurons, but not for sinusoidally coupled phase oscillators, is the appearance of stable intermediate values $w_{2} \in\left[w_{\min }, w_{\max }\right]$; see Fig. 8 . 

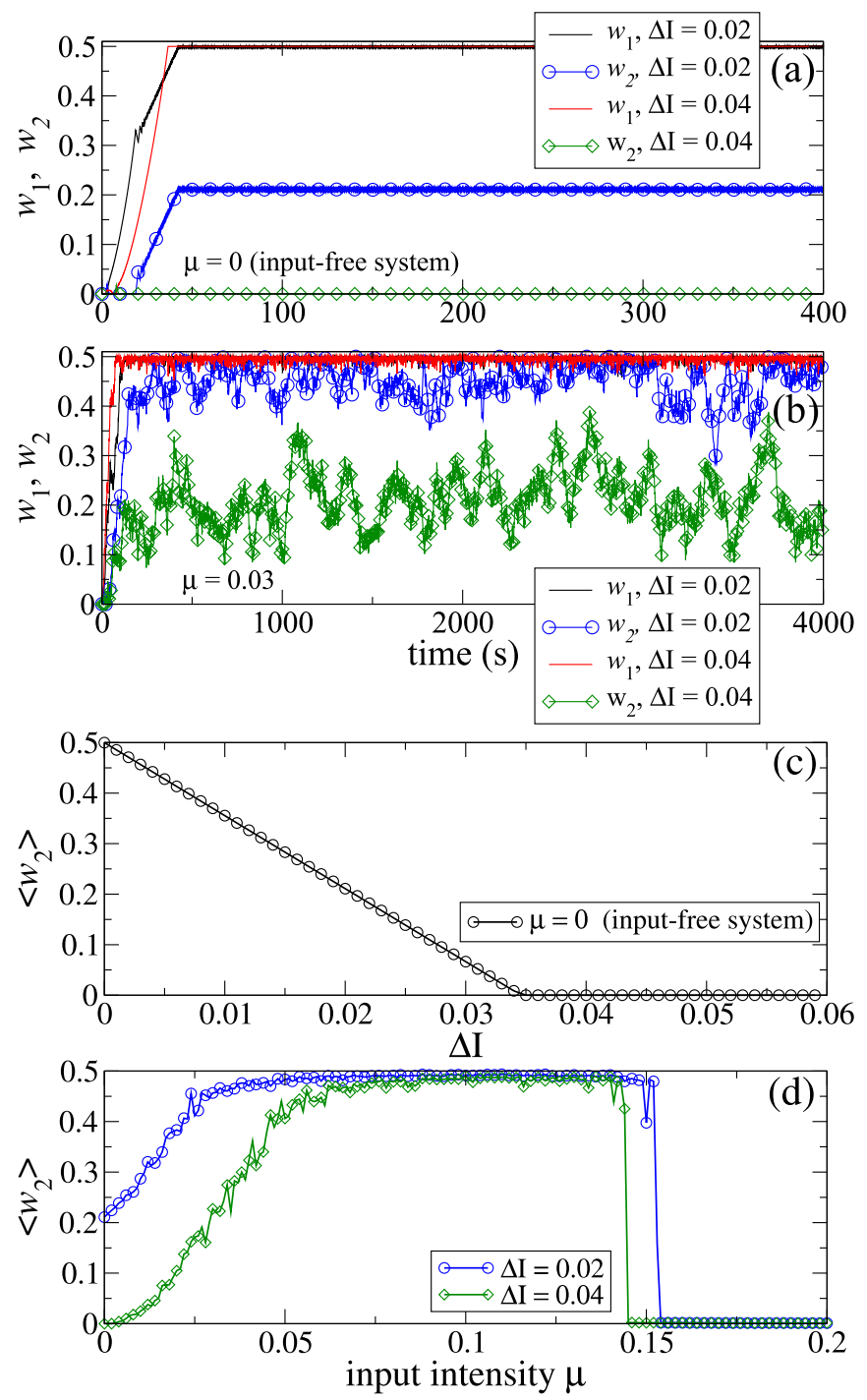

FIG. 11. Time courses of the synaptic weights $w_{1}$ and $w_{2}$ in system (25) for (a) input-free case with $\mu=0$ and (b) neurons perturbed by an independent random synaptic input of intensity $\mu=0.03$ and different parameter mismatches $\Delta I$ as indicated in the legends. As initial state, the uncoupled regime $w_{1}(0)=w_{2}(0)=0.0$ was taken. Plots (c) and (d) show the time-averaged synaptic weight $\left\langle w_{2}\right\rangle$ in system (25) vs (c) parameter mismatch $\Delta I$ for the input-free case $\mu=0$, (d) input intensity $\mu$ for different $\Delta I$ as indicated in the legend. The other synaptic weight $w_{1} \approx w_{\max }=0.5$ [cf. (a) and (b)].

For small parameter mismatch $\Delta I$ these intermediate values can already be found in the noise-free case $\mu=0$ [Figs. 11(a) and 11(c)]. They mediate small values of $w_{2} \approx 0$ for large $\Delta I$ and large (maximal) values of $w_{2}$ for identical oscillators, i.e., $\Delta I=0$ [Fig. 11(c)], where the symmetric bidirectional coupling is stable also in the input-free case. The intermediate values of $w_{2}$ extend to the two-parameter plane $(\Delta I, \mu)$ for finite noise intensity $\mu>0$ [Figs. 11(b) and 11(d)]. In this section we consider the emergence of intermediate values of $w_{2}$ for the cases $\mu=0$ and $\mu>0$. This phenomenon has also been verified for the reversal potential $V_{r}=0 \mathrm{mV}$ as well as for the multiplicative update rule with soft bounds; see Appendix.

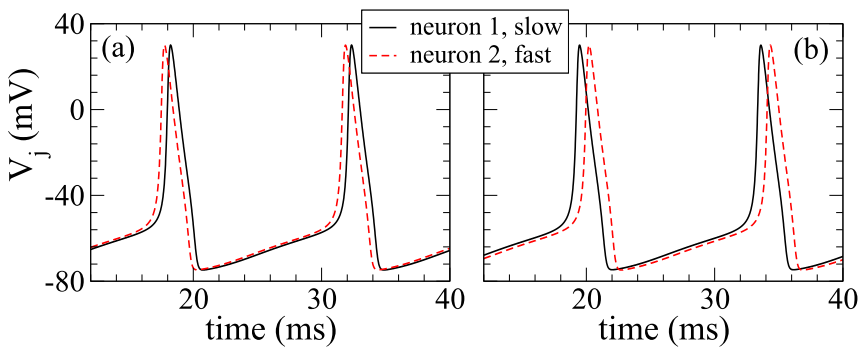

(c)
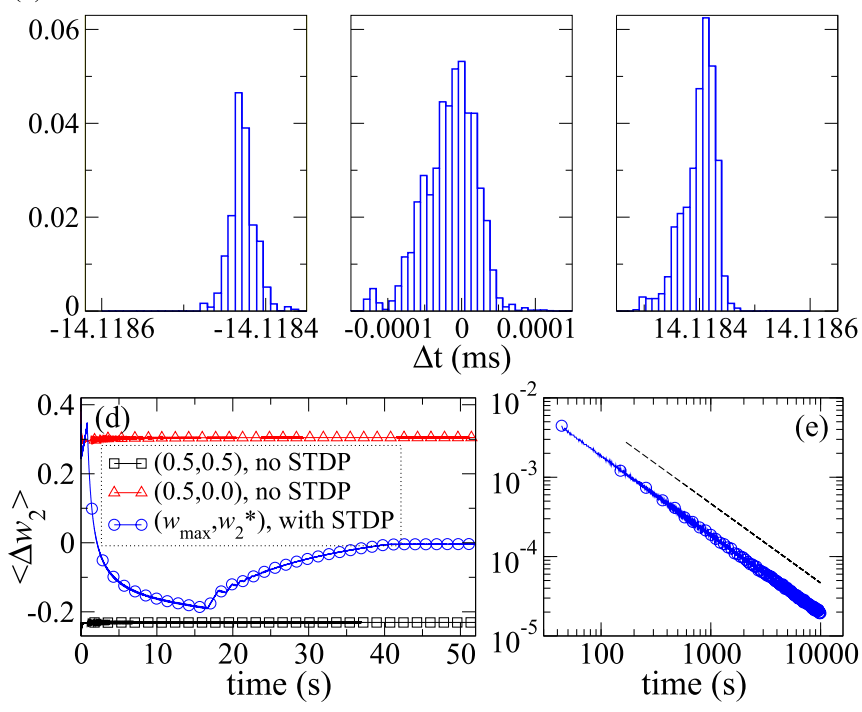

FIG. 12. Neuronal dynamics in the input-free $(\mu=0)$ system (25) for (a) and (b) fixed synaptic weights (STDP is off) $\left(w_{1}, w_{2}\right)=$ $(0.5,0.5)$ and $\left(w_{1}, w_{2}\right)=(0.5,0.0)$, respectively, and (c) adaptive synaptic weights (STDP is on) approaching the state $\left(w_{1}, w_{2}\right)=$ $\left(w_{\max }, w_{2}^{*}\right)$ with $w_{2}^{*} \approx 0.21$ due to STDP; see Fig. 11 (a) for $\Delta I=0.02$. In plots (a) and (b) the time courses of the membrane potentials $V_{j}$ of the neurons are shown, and (c) depicts the distribution density of the spiking time difference $\Delta t=t_{\text {post }}-t_{\text {pre }}$ for the postsynaptic neuron 2 contributing to the update of synaptic weight $w_{2}$. (d) Dynamics of the average update $\left\langle\Delta w_{2}\right\rangle$ (see text for definition) for the conditions of plots (a)-(c), as indicated in the legend. (e) Log-log plot of $\left\langle\Delta w_{2}\right\rangle$ for adaptive synaptic weights [blue circles in plot (d), corresponding to conditions of plot (c) and Fig. 11(a) for $\Delta I=0.02$ ]. The black dashed line has a slope -1 and is given for comparison. Parameters $\Delta I=0.02$ and $\mu=0$.

Let us first turn the STDP off and fix the coupling $w_{1}=$ $w_{\max }=0.5$ and $w_{2}=0$ or $w_{2}=w_{\max }$ for $\Delta I=0.02$ and $\mu=0$. We find that for both coupling cases the neurons are well locked to each other, where the time lag between spikes is well separated from zero [Figs. 12(a) and 12(b)]. In the case of bidirectional coupling $\left(w_{1}, w_{2}\right)=(0.5,0.5)$ the spikes of the fast neuron 2 advance those of the slow neuron 1 [Fig. 12(a)]. In the case of unidirectional coupling $\left(w_{1}, w_{2}\right)=(0.5,0.0)$, where the fast neuron 2 drives the slow neuron 1, the spiking sequence is reversed such that the spikes of fast neuron 2 follow the spikes of slow neuron 1 [Fig. 12(b)]. The observed reversing of the spike timing differs from the dynamics of the phase oscillators (1) with sinusoidal coupling function $g(\varphi)=\sin (\varphi)$. There the fast oscillator always advances the slow oscillator in the phase-locked regime, irrespectively of the coupling configuration. 
The spike timing illustrated in Figs. 12(a) and 12(b) leads to that, due to STDP, the synaptic weight $w_{2}$ must increase when starting from the unidirectional coupling $\left(w_{1}, w_{2}\right)=$ $(0.5,0.0)$ and decrease when starting from the bidirectional coupling $\left(w_{1}, w_{2}\right)=(0.5,0.5)$. Indeed, the average update of $w_{2}$ calculated as $\left\langle\Delta w_{2}\right\rangle(t)=N_{\mathrm{sp}}^{-1} \sum_{j=1}^{N_{\mathrm{sp}}} \Delta w_{j}$, where $\Delta w_{j}$ is the corresponding update of $w_{2}$ at the $j$ th spike, and $N_{\mathrm{sp}}$ is the number of spikes in the time interval $[0, t]$, converges to 0.3 for the fixed unidirectional coupling [Fig. 12(d), red triangles] and to -0.23 for the fixed bidirectional coupling [Fig. 12(d), black squares]. When STDP is turned on, $w_{2}$ approaches $w_{2}^{*} \approx 0.21$ [Fig. 11(a)], where an exchange between the two spiking regimes illustrated in Figs. 12(a) and 12(b) takes place. In this regime of $\left(w_{1}, w_{2}\right)=\left(w_{\max }, w_{2}^{*}\right)$ a potentiation of $w_{2}$ over $w_{2}^{*}$ leads to the spiking ordering as in Fig. 12(a), which induces a depression of $w_{2}$. On the other hand, for smaller $w_{2}$ the spiking ordering becomes as in Fig. 12(b), which causes a potentiation of $w_{2}$. The synaptic weights slightly fluctuate around the above state [Fig. 11(a) for $\Delta I=0.02$ ], and the spiking time difference $\Delta t=t_{\text {post }}-t_{\text {pre }}$ between the postsynaptic neuron 2 and the presynaptic neuron 1 gets distributed around zero [Fig. 12(c)], where the neurons fire spikes in a mixed order even without a noise input. In this coupling regime, the average update $\left\langle\Delta w_{2}\right\rangle$ converges to zero as $t^{-1}$ [Figs. 12(d) and 12(e), blue circles], which indicates a saturation of $w_{2}$ at the intermediate value $w_{2}^{*}$.

For a larger parameter mismatch, for instance, $\Delta I=0.04$, the input-free $(\mu=0)$ neurons fire in the same well-defined order for both fixed bidirectional and unidirectional couplings, where the fast neuron advances the slow one. An example of the distribution density of the spiking time difference $\Delta t$ for fixed $\left(w_{1}, w_{2}\right)=(0.5,0)$ and vanishing input $(\mu=0)$ is illustrated in Fig. 13(a) (black filled histogram). We found that the average update $\left\langle\Delta w_{2}\right\rangle$ converges to negative values -0.22 for $\left(w_{1}, w_{2}\right)=(0.5,0.5)$ and -0.24 for $\left(w_{1}, w_{2}\right)=$ $(0.5,0.0)$. Therefore, due to STDP, the input-free system (25) approaches the unidirectional coupling regime $\left(w_{1}, w_{2}\right)=$ $\left(w_{\max }, 0\right)$ [Fig. 11(a) for $\Delta I=0.04$ ]

With the noise input, however, the synaptic weight $w_{2}$ can be potentiated. For example, for the fixed synaptic weight (no STDP) $\left(w_{1}, w_{2}\right)=(0.5,0.5)$ and noise intensity $\mu=0.03$, the spiking time difference $\Delta t$ gets broadly distributed [Fig. 13(a), red hatched histogram], nevertheless, the average update $\left\langle\Delta w_{2}\right\rangle$ of $w_{2}$ still remains negative [Fig. 13(c), red triangles]. On the other hand, for the fixed unidirectional coupling $\left(w_{1}, w_{2}\right)=(0.5,0)$, which is stable under STDP for vanishing noise [Fig. 11(a)], the random input of the same intensity leads to such a neuronal dynamics and distribution of the spiking time difference [Fig. 13(a), blue empty histogram] that the average update $\left\langle\Delta w_{2}\right\rangle$ becomes positive [Fig. 13(c), blue squares]. Therefore, when STDP is switched on, $w_{2}$ approaches intermediate values because it will be potentiated if it approaches zero and will be depressed if it approaches $w_{\max }$. For the considered parameters, the synaptic weights were found to fluctuate around $\left(w_{\max }, w_{2}^{*}\right)$ with $w_{2}^{*}=\left\langle w_{2}\right\rangle \approx$ 0.2 [Fig. 11(b), green circles]. In this coupling regime, the distribution of $\Delta t$ [Fig. 13(d)] interpolates between the two noise-induced distributions of $\Delta t$ in Fig. 13(a) [blue empty and red hatched histograms] for the limiting cases of $w_{2}=0$ and $w_{2}=w_{\max }$, and the average update $\left\langle\Delta w_{2}\right\rangle$ of $w_{2}$ converges (a)
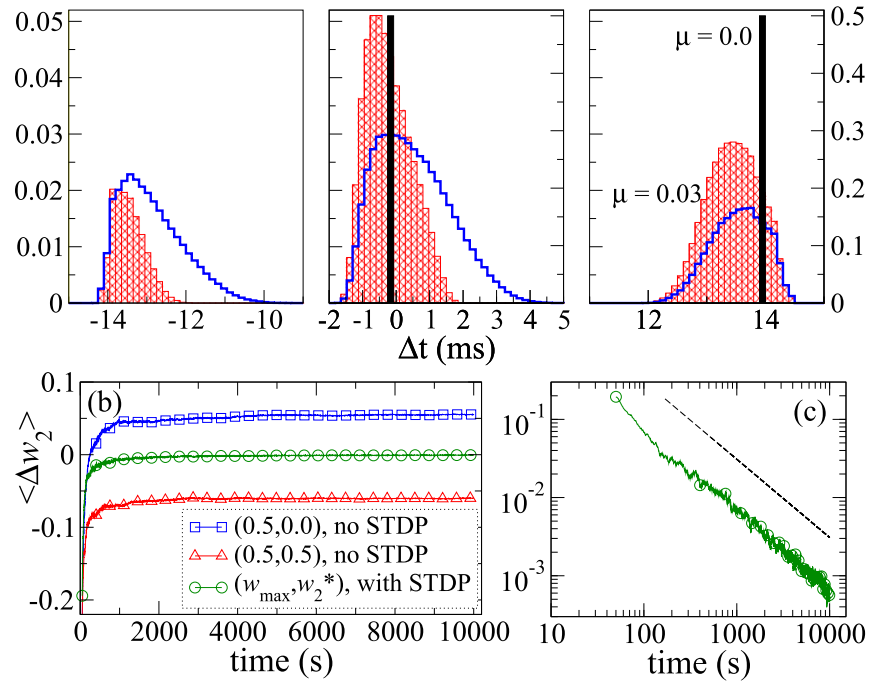

(d)
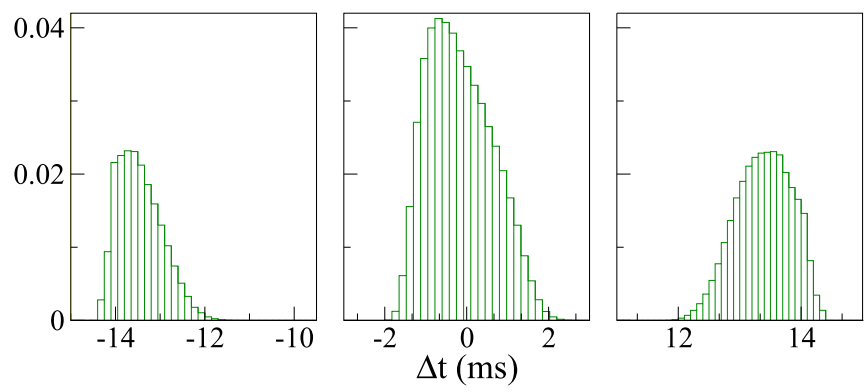

FIG. 13. Impact of the random input on the neuronal dynamics of system (25). (a) Distribution densities of the spiking time difference $\Delta t=t_{\text {post }}-t_{\text {pre }}$ for the postsynaptic neuron 2 contributing to the update of synaptic weight $w_{2}$ for the fixed coupling (STDP is off) $\left(w_{1}, w_{2}\right)=(0.5,0)$ (blue empty histogram) and $\left(w_{1}, w_{2}\right)=(0.5,0.5)$ (red hatched histogram) and input intensity $\mu=0.03$. The distribution of $\Delta t$ for $\left(w_{1}, w_{2}\right)=(0.5,0)$ and vanishing input $(\mu=0)$ is depicted by black filled histogram (scaling on the right vertical axis). (b) Dynamics of the average update $\left\langle\Delta w_{2}\right\rangle$ for the two conditions $\left(w_{1}, w_{2}\right)=(0.5,0)$ and $\left(w_{1}, w_{2}\right)=(0.5,0.5)$ of plot (a) with random input and fixed coupling (blue squares and red triangles) and adaptive synaptic weights (green circles) as indicated in the legend. (c) Log-log plot of $\left\langle\Delta w_{2}\right\rangle$ for the case when STDP is on [green circles in plot (b), corresponding to conditions of Fig. 11(b) for $\Delta I=0.04]$. The black dashed line has a slope -1 and is given for comparison. (b) Distribution density of $\Delta t$ for adaptive synaptic weights (STDP is on), where $\left(w_{1}, w_{2}\right)$ fluctuates around $\left(w_{\max }, w_{2}^{*}\right)$ with $w_{2}^{*} \approx 0.2$ [Fig. 11(b) for $\Delta I=0.04]$ corresponding to the case depicted by green circles in plots (b) and (c). Parameters $\Delta I=0.04$ and $\mu=0.03$.

to zero as $t^{-1}$ [Figs. 13(c) and 13(d), green circles]. For larger noise, $w_{2}$ may further increase [Fig. 11(d)]. The above example illustrates the mechanism of the noise-induced potentiation of coupling in systems with STDP.

\section{CONCLUSION}

In the first part of the paper, we developed an analytical approach to predict the slow dynamics of coupling weights in a pair of coupled oscillatory neurons modeled by phase oscillators subject to noise and STDP. Our main result is the 
reduction of the stochastic dynamics to a two-dimensional system of ordinary differential equations which describe the coupling dynamics. It is worthwhile to mention two assumptions again. First, in order to transform the STDP rule to a globally valid PDDP rule the relative detuning $\Delta \omega / \Omega$ of the natural frequencies has to be small. Second, the coupling dynamics have to be slow enough to render valid the averaging of the FPE and thereby enable us to obtain Eq. (18). Under these assumptions the proposed technique is very general and can be applied to arbitrary coupling and plasticity functions.

The numerical bifurcation analysis for an example with sinusoidal coupling and an exponential PDDP rule revealed a possibility of multistability of the uncoupled, the unidirectionally, and the bidirectionally coupled states and the presence of self-organized resistance to noise.

It is certain that the PDDP model yields qualitatively equivalent results in many situations though there are some features of the STDP case which may not be captured. For instance the values of the natural frequencies of the phase oscillators do not enter the calculations. Merely the frequency detuning $\Delta \omega=\omega_{2}-\omega_{1}$ appears in the equations. However, the natural frequencies do show up independently when an STDP rule is applied, i.e., the spike time differences are taken into account for the update of the coupling strength. For instance, this is the reason why the stability of the uncoupled state is independent of $\mu$ and $\Delta \omega$ in the PDDP model, while it may change with a variation of $\mu$ and $\Delta I$ in the Hodgkin-Huxley model [Fig. 8(a)].

The results obtained for the phase oscillators are qualitatively confirmed for a system of two spiking HodgkinHuxley neurons; see Sec. VI. In particular, we show that a random independent synaptic input leads to the emergence of bidirectional coupling between neurons, which strengthens the overall connectivity in the system as observed for large neuronal ensembles [18]. As for the phase oscillators, the noisy input can induce a multistability in coupled neurons and switching between different stable coupling regimes. In the case of Hodgkin-Huxley neurons all four different stable states (combinations of $w_{j} \in\left\{0, w_{\max }\right\}$ ) can coexist [cf. Fig. 10(c)], while for the phase model with $g(\varphi)=\sin (\varphi)$ a coexistence of both unidirectionally coupled states is impossible. Moreover, for a certain parameter range we observe for the coupled Hodgkin-Huxley neurons that the average value of coupling strengths saturates at intermediate values, i.e., $\left\langle w_{2}\right\rangle \approx w^{*}$, where $w^{*}$ is neither close to zero nor to the maximal coupling strength. Such states of intermediate asymptotic coupling strengths, as well as a multistability of both unidirectionally coupled states, can also be observed for coupled phase oscillators with coupling functions $g(\varphi) \neq \sin (\varphi)$ (data not shown). In this context, it can be interesting to explore the validity of the proposed technique for the phase reductions of different neuron models in detail.

Our findings demonstrate that the reported noise-induced effects are rather general phenomena and take place for very different models of the intrinsic dynamics (phase oscillators or conductance-based model neurons), plasticity rules (PDDP or STDP), coupling type (based on the phase differences or chemical synaptic coupling), and random perturbations (noise or random synaptic input). The reported results are valid under the natural assumption that the coupling induces a synchronization, which however may not be the case, for instance, for integrate-and-fire neurons or when the excitatory synapses are too slow [49,50]. For analytical derivations and numerical simulations we used Gaussian noise for random input. The results are expected to be preserved for input with independent Poisson spike trains [18].

Our results contribute to a better understanding of stimulation-induced changes of collective neuronal dynamics, especially of neuronal synchronization. Apart from its clinical relevance [see Introduction], our findings might be relevant to other neuroscientific issues, e.g., for the relation between intrinsic dynamics and stimulus responses [51-53]. We have demonstrated that the firing pattern of a fundamental neuronal building block, two neurons connected through plastic synapses, is not just a simple reflection of the stimulus' properties. In fact, already in the presence of a putatively simple stimulus, uncorrelated noise, a variety of different dynamical regimes might be present, depending on characteristic parameters intrinsic to the neurons involved. In networks of phase oscillators with constant coupling it was shown that the response to pulsatile stimuli may crucially depend on the coupling mechanism [54]. Hence, the interplay between ongoing input, caused by other neurons and modulated by ongoing sensory input, and pulsatile (sensory) stimuli may lead to a substantial variety of stimulus-induced responses as observed experimentally [51-53]. Accordingly, in future studies one could additionally take into account pulsatile stimuli to study the repertoire of transient stimulusinduced responses and their relation to the initial spontaneous state.

\section{ACKNOWLEDGMENTS}

We thank the German Research Foundation (DFG) for financial support in the framework of the Collaborative Research Center 910 (L.L.), the European Research Council (ERC-2010-AdG 267802, Analysis of Multiscale Systems Driven by Functionals), and the Clinical Research Unit 219 (TA203/4-1 to P.A.T.).

L.L. and O.V.P. contributed equally to this work.

\section{APPENDIX: MULTIPLICATIVE UPDATE WITH SOFT BOUNDS}

In this study we considered the STDP rule with additive update of synaptic weights $w_{i} \rightarrow w_{i}+\delta \Delta w(\Delta t)$ with socalled hard bounds, where $w_{i} \in\left[w_{\min }, w_{\max }\right]$ is set to $w_{\min }$ or to $w_{\max }$ if it is depressed to a lower value than $w_{\min }$ or potentiated over $w_{\max }$, respectively $[2,16,18,44]$. Another approach consists of a multiplicative (state-dependent) update rule, where the coupling weights are changed relative to their current values $w_{i} \rightarrow w_{i}+\left(w_{M}-w_{i}\right)|\delta \Delta w(\Delta t)|$, where $w_{M}=$ $w_{\max }$ or $w_{M}=w_{\min }$ depending on whether the coupling update $\delta \Delta w(\Delta t)$ is positive (potentiation) or negative (depression), respectively $[15,19,20,42,45,46]$. For the multiplicative update rule the potentiation (depression) gradually weakens when the synaptic weights approach upper (lower) bound, which makes them confined to the interval $\left[w_{\min }, w_{\max }\right]$. 
For the same parameters of the STDP function (see Sec. VI) the input-free $(\mu=0) \mathrm{HH}$ neurons (25) with multiplicative STDP rule demonstrate a similar behavior of the synaptic weights as for the additive update; see Fig. 14(a) and compare it with Fig. 11(c). For large parameter detuning $\Delta I$, the neurons are unidirectionally coupled, where the fast neuron 2 drives the slow neuron 1 . For small $\Delta I$ we observe intermediate values of $w_{2}$ with large values of $w_{1}$. Identical neurons become symmetrically bidirectionally coupled as for the additive update, although the synaptic weights deviate from the maximal value $w_{\max }$ [Fig. 14(a)].

The independent random input potentiates the coupling from slow neuron to fast neuron and depresses the coupling in the opposite direction [Figs. 14(b) and 14(c)]. For large enough noise the neurons become bidirectionally coupled as for the additive update, but the synaptic weights saturate at an intermediate value. As for the additive update rule, the multiplicative update also enhances the mean coupling $\left(w_{1}+w_{2}\right) / 2$ when the noise strength increases [Figs. 14(b) and 14(c), blue triangles]. This effect becomes more pronounced for large neuronal ensembles as reported in Ref. [18]. However, even for strong noise the neurons remain strongly coupled in spite of the asynchronous neuronal firing [Fig. 14(d)]. As compared to the additive STDP rule, the considered multiplicative update can hardly differentiate between synchronized and desynchronized neuronal dynamics by the extent of connectivity: for both regimes the multiplicative STDP rule leads to large mean coupling in the neuronal population (Fig. 14), see also Ref. [18]. Another essential difference between the additive and multiplicative STDP rules is that we did not observe any multistability of diverse coupling regimes for the latter case. For the considered parameters and for different initial conditions the connectivity converges to the same state illustrated in Fig. 14. In this sense the dynamics of coupling governed by the multiplicative STDP rule appears to be much simpler.
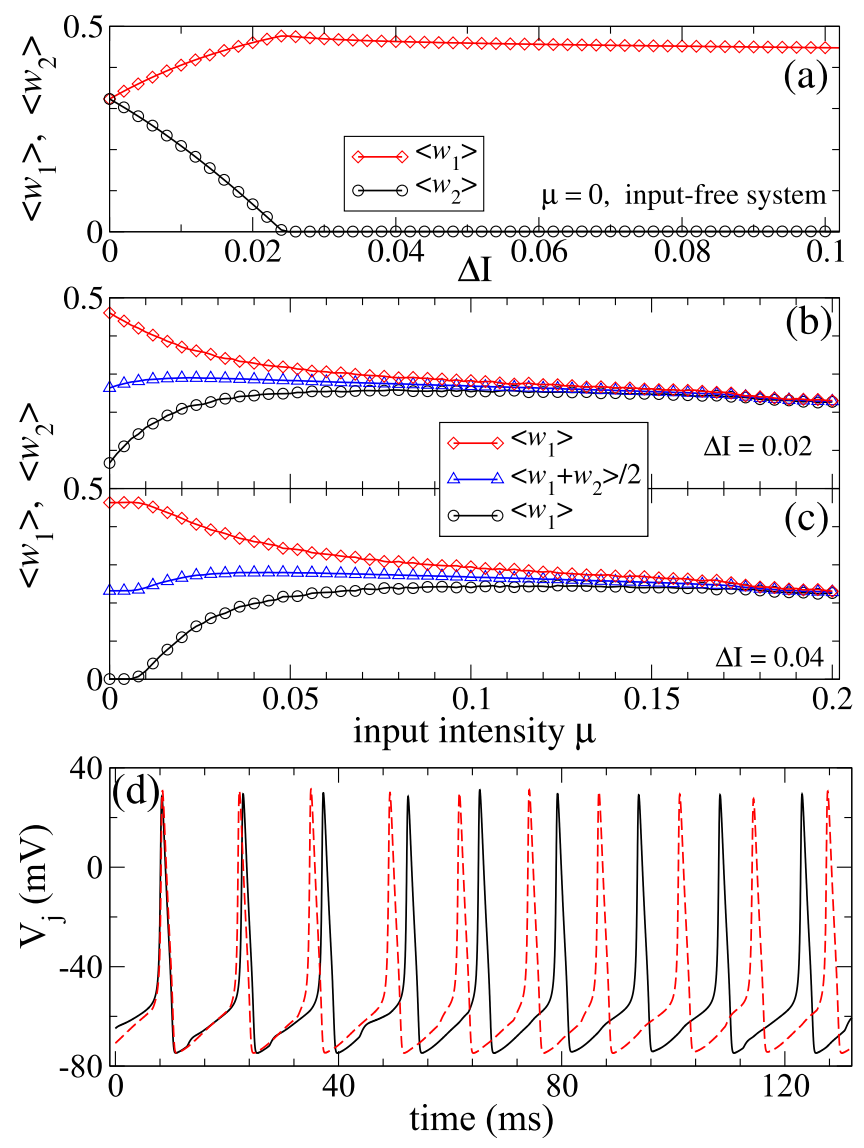

FIG. 14. Time-averaged synaptic weights in system (25) for the multiplicative update rule with soft bounds vs (a) parameter mismatch $\Delta I$ for the input-free case $\mu=0$ and (b) and (c) input intensity $\mu$ for different $\Delta I$ as indicated in the plots. (d) Time courses of the membrane potentials of the HH neurons (25) for $\Delta I=0.02$, noise intensity $\mu=0.2$, and coupling regime from plot (b). Parameter $V_{r}=0 \mathrm{mV}$.
[1] D. O. Hebb, The Organization of Behavior: A Neuropsychological Approach (John Wiley \& Sons, New York, 1949).

[2] W. Gerstner, R. Kempter, J. L. van Hemmen, and H. Wagner, Nature (London) 383, 76 (1996).

[3] H. Markram, J. Lübke, M. Frotscher, and B. Sakmann, Science 275, 213 (1997).

[4] G.-q. Bi and M.-m. Poo, J. Neurosci. 18, 10464 (1998).

[5] L. F. Abbott and S. B. Nelson, Nat. Neurosci. 3, 1178 (2000).

[6] D. E. Feldman, Neuron 27, 45 (2000).

[7] G.-q. Bi and M.-m. Poo, Annu. Rev. Neurosci. 24, 139 (2001).

[8] W. Gerstner and W. M. Kistler, Spiking Neuron Models: Single Neurons, Populations, Plasticity (Cambridge University Press, Cambridge, UK, 2002).

[9] G. M. Wittenberg and S. S.-H. Wang, J. Neurosci. 26, 6610 (2006).

[10] N. Caporale and Y. Dan, Annu. Rev. Neurosci. 31, 25 (2008).

[11] C. Clopath, L. Busing, E. Vasilaki, and W. Gerstner, Nat. Neurosci. 13, 344 (2010).
[12] D. Shulz and D. Feldman, in Neural Circuit Development and Function in the Brain, edited by J. L. Rubenstein and P. Rakic (Academic, Oxford, 2013), pp. 155-181.

[13] P. A. Tass and M. Majtanik, Biol. Cybern. 94, 58 (2006).

[14] N. Masuda and H. Kori, J. Comput. Neurosci. 22, 327 (2007).

[15] T. Aoki and T. Aoyagi, Phys. Rev. E 84, 066109 (2011).

[16] A. Knoblauch, F. Hauser, M.-O. Gewaltig, E. Körner, and G. Palm, Front. Comput. Neurosci. 6, 55 (2012).

[17] M. Bayati and A. Valizadeh, Phys. Rev. E 86, 011925 (2012).

[18] O. V. Popovych, S. Yanchuk, and P. A. Tass, Sci. Rep. 3, 2926 (2013).

[19] P. Seliger, S. C. Young, and L. S. Tsimring, Phys. Rev. E 65, 041906 (2002).

[20] Y. L. Maistrenko, B. Lysyansky, C. Hauptmann, O. Burylko, and P. A. Tass, Phys. Rev. E 75, 066207 (2007).

[21] Q. Ren and J. Zhao, Phys. Rev. E 76, 016207 (2007).

[22] V. Chandrasekar, J. H. Sheeba, B. Subash, M. Lakshmanan, and J. Kurths, Physica D 267, 36 (2014). 
[23] O. V. Popovych, M. N. Xenakis, and P. A. Tass, PLoS ONE 10, e0117205 (2015).

[24] P. Tass and C. Hauptmann, Nonlin. Phenom. Complex Syst. 9, 298 (2006).

[25] C. Hauptmann and P. A. Tass, Biosystems 89, 173 (2007).

[26] P. A. Tass and C. Hauptmann, Restor. Neurol. Neurosci. 27, 591 (2009).

[27] C. Hauptmann and P. A. Tass, J. Neural Eng. 6, 016004 (2009).

[28] O. V. Popovych and P. A. Tass, Front. Hum. Neurosci. 6, 58 (2012).

[29] P. A. Tass, L. Qin, C. Hauptmann, S. Doveros, E. Bezard, T. Boraud, and W. G. Meissner, Ann. Neurol. 72, 816 (2012).

[30] P. A. Tass, I. Adamchic, H.-J. Freund, T. von Stackelberg, and C. Hauptmann, Rest. Neurol. Neurosci. 30, 137 (2012).

[31] P. A. Tass and O. V. Popovych, Biol. Cybern. 106, 27 (2012).

[32] A. N. Silchenko, I. Adamchic, C. Hauptmann, and P. A. Tass, Neuroimage 77, 133 (2013).

[33] I. Adamchic, T. Toth, C. Hauptmann, and P. A. Tass, Hum. Brain Mapp. 35, 2099 (2014).

[34] I. Adamchic, C. Hauptmann, U. B. Barnikol, N. Pawelczyk, O. Popovych, T. T. Barnikol, A. Silchenko, J. Volkmann, G. Deuschl, W. G. Meissner et al., Mov. Disorders 29, 1679 (2014).

[35] H. Sakaguchi, Prog. Theor. Phys. 79, 39 (1988).

[36] H. Nakao, K. Arai, and Y. Kawamura, Phys. Rev. Lett. 98, 184101 (2007).

[37] W. Kurebayashi, K. Fujiwara, and T. Ikeguchi, Europhys. Lett. 97, 50009 (2012).

[38] C. Ly and G. Ermentrout, J. Comput. Neurosci. 26, 425 (2009).
[39] C. W. Gardiner, Handbook of Stochastic Methods (Springer, Berlin, 1985), Vol. 3.

[40] T. Aoki and T. Aoyagi, Phys. Rev. Lett. 102, 034101 (2009).

[41] M. Li, S. Guan, and C.-H. Lai, New J. Phys. 12, 103032 (2010).

[42] A. Morrison, M. Diesmann, and W. Gerstner, Biol. Cybern. 98, 459 (2008).

[43] R. C. Froemke and Y. Dan, Nature (London) 416, 433 (2002).

[44] S. Song, K. Miller, and L. Abbott, Nat. Neurosci. 3, 919 (2000).

[45] J. Rubin, D. D. Lee, and H. Sompolinsky, Phys. Rev. Lett. 86, 364 (2001).

[46] R. Gütig, R. Aharonov, S. Rotter, and H. Sompolinsky, J. Neurosci. 23, 3697 (2003).

[47] A. Hodgkin and A. F. Huxley, J. Physiol. 117, 500 (1952).

[48] D. Hansel, G. Mato, and C. Meunier, Europhys. Lett. 23, 367 (1993).

[49] C. Van Vreeswijk, L. F. Abbott, and G. B. Ermentrout, J. Comput. Neurosci. 1, 313 (1994).

[50] D. Hansel, G. Mato, and C. Meunier, Neural Comput. 7, 307 (1995).

[51] J. Mast and J. D. Victor, Electroencephal. Clin. Neurophysiol. 78, 389 (1991).

[52] A. Arieli, A. Sterkin, A. Grinvald, and A. Aertsen, Science 273, 1868 (1996).

[53] J. Fiser, C. Chiu, and M. Weliky, Nature (London) 431, 573 (2004).

[54] P. Tass, Phase Resetting in Medicine and Biology. Stochastic Modelling and Data Analysis, Springer Series in Synergetics (Springer, Berlin, 1999). 